Preprint KUL-TF-96/4

NIKHEF-96-009

hep-th/9603012

March 96

\title{
The BPHZ renormalised BV master equation and Two-loop Anomalies in Chiral Gravities
}

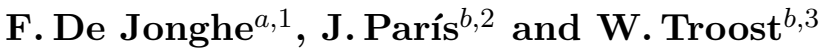 \\ ${ }^{a}$ NIKHEF/FOM, Postbus 41882, 1009 DB Amsterdam, The Netherlands \\ ${ }^{b}$ Instituut voor Theoretische Fysica, K.U.Leuven \\ Celestijnenlaan 200D, B-3001 Leuven, Belgium
}

\begin{abstract}
Anomalies and BRST invariance are governed, in the context of Lagrangian Batalin-Vilkovisky quantization, by the master equation, whose classical limit is $(S, S)=0$. Using Zimmerman's normal products and the BPHZ renormalisation method, we obtain a corresponding local quantum operator equation, which is valid to all orders in perturbation theory. The formulation implies a calculational method for anomalies that is useful also outside this context and that remains completely within regularised perturbation theory. It makes no difference in principle whether the anomaly appears at one loop or at higher loops. The method is illustrated by computing the one- and two-loop anomalies in chiral $W_{3}$ gravity.
\end{abstract}

\footnotetext{
E-mail : t54@nikhef.nl

${ }^{2}$ E-mail: jordi.paris@fys.kuleuven.ac.be

${ }^{3}$ Onderzoeksleider N.F.W.O., Belgium

E-mail: walter.troost@fys.kuleuven.ac.be
} 


\section{Introduction}

The most comprehensive method for treating gauge theories is at present the method of Batalin and Vilkovisky (BV). It unifies in one framework the description of gauge invariance, the selection of physical observables, the gauge fixing, BRS methods, the introduction of ghosts (and antighosts, if wanted), etc. All this is achieved irrespective of whether the gauge algebra is a relatively simple one (as for nonabelian gauge theories) or a more complicated one, closing only upon using the equations of motion (a BRST charge that is nilpotent only on shell), as often occurs in supergravity and in string theories.

Whereas over the years the quantum BRS treatment of gauge theories [1] has met with many successes [2], the quantum theory in the BV scheme is less developed. Indeed, the remarks above strictly apply to the classical theory. For many considerations, including some quantum questions, like the classifications of one loop anomalies for example, one practically needs use only of the classical theory: interesting results follow by considering that in the quantum theory the anomalies have to satisfy a certain condition and by solving its classical version (the Wess-Zumino consistency condition). A full quantum analysis however requires a more careful treatment. It is rather easy to give formal arguments, and in fact they were already given in the founding papers on the subject [3]; but when one goes deeper into the matter, taking seriously the occurrence of the well-known divergences, one realises that the literature is defective. This paper addresses this problem head-on.

In the BV setup, the gauge invariance at the classical level is expressed by the so-called master equation, $(S, S)=0$. Here $S$ is the extended classical action, which consists of the classical action (including ghosts and antighosts), source terms for the BRS-transformation laws, and some supplementary terms if the gauge algebra is open. On the quantum level, BRST invariance can be transcribed as $(\Gamma, \Gamma)=0$, where $\Gamma$ is the generating functional of the one-particle irreducible graphs, or the effective action, evaluated in the presence of the above mentioned source terms. Anomalies consist then in a violation of this quantum equation. In the BRS context, this question was developed making extensive use of the formulation of the quantum theory on the level of the effective action. This development can of course be transcribed to the BV formalism. However, the question arises also how this translates in the quantum theory to the local level, i.e. to the level of the action. Formal manipulations modify the classical master equation into $\left(S_{q}, S_{q}\right)=2 i \hbar \Delta S_{q}$, where the quantum action possibly contains terms that are explicitly of higher order in $\hbar, S_{q}=S+\mathcal{O}(\hbar)$ and $\Delta$ is a second order functional differential operator where the functional derivatives are at the same space-time point. If $S_{q}$ is a local action, this operation leads to $\delta(0)$, it is ill-defined. The source of the problem is of course the same as always in local quantum field theory, namely the coincidence of space-time points. A regularisation scheme should solve it.

Efforts in this direction include 4 where the method of Pauli and Villars has been applied to this question. This proposal works well on the one loop level, but it is not very clear how to extend this type of calculation to higher loops. In a less conventional approach, the use of nonlocal regularisation was suggested [5, 6], providing a method valid also for higher order loops. In [7], an analysis of the quantum aspects of BV using dimensional regularization is presented.

The interest in a full local quantum treatment is not a purely conceptual one. It is also of importance for the question of anomalies appearing in higher loops. Indeed, in some theories, contrary to the situation in nonabelian gauge theories [8], anomalies can arise at the multiple loop level. This is notably the case in extended theories of two dimensional gravity, so-called $W$-gravities. The presence of a two-loop anomaly in $W_{3}$-gravity, for example, is ascertained through the use of the operator product expansions of two dimensional conformal field theory. Whereas this is certainly a valid method, it leaves a gap. Namely, rather than deriving these operator product expansions from a proper treatment of the renormalised quantum field theory 
that underlies them, their validity is mostly assumed, based on algebraic and symmetry considerations. Clearly, a complete treatment of higher loop anomalies in renormalised perturbative quantum field theory is lacking.

The purpose of the present paper is to formulate and derive the basic local equations governing the BV method on the level of renormalised perturbation theory. On a more practical level, we will provide a proper method, always within conventional renormalised perturbation theory, to compute higher loop anomalies. We will use the method of Bogoliubov, Parasiuk, Hepp and Zimmerman (BPHZ) to achieve this purpose. Not only is this method of impeccable standing, but also we will find that it is in fact well-suited to put the quantum master equation of the BV formalism on a solid foundation. In fact, that it is suited for computing anomalies (in global symmetries) was already realised long ago (see [9]). Treatments of non-abelian gauge theories, using the same operator methods, on the level of the effective action, have of course played an important role in firmly establishing their renormalisability (after the dimensional regularisation treatment of [10]). An overview of this and other developments that goes together well with the BV scheme can be found in 11 .

This paper should not be read as a complete account of the way the BV formalism and the BPHZ method work together to provide a full account, in renormalised quantum field theory, of all possible higher loop anomalies. Rather it should be viewed as the onset of a program. It does provide the basic equations and explicit examples. Working out the detailed consequences in the most general situations is left for the future. As far as the method to compute BRST anomalies is concerned, it can be stripped of its BV dressing and applied outside this context as well.

We have organized the paper as follows. In section 2 we recall the basics of the BatalinVilkovisky method, the relationship of the quantum master equation with the occurrence of anomalies, and the Zinn-Justin equation, thus setting the stage for a more precise formulation of what we want to achieve. Section 3 starts with an extensive discussion of renormalisation schemes in the BV setting, thus argueing for the use of the BPHZ method. The basics of this method, including the manifestation of anomalies (in particular the chiral anomaly) are then recalled, and some aspects of the massless case are presented from an angle that complements the existing literature. Section 1 combines the previous developments into a general derivation of the master equation in the BPHZ framework. The corresponding treatment for the concrete case of $W_{2}$-gravity in section 5 serves as an illustration of the more abstract treatment of the previous sections, and also as a warm-up for the treatment of $W_{3}$ gravity in section 6 wherein, after quickly giving the one-loop anomaly, we come to the complete computation of the twoloop anomaly of $W_{3}$. Three appendices collect some formulas on BPHZ renormalized one-loop integrals for $2 D$ chiral gravities, supply the complete one-loop anomaly for $W_{3}$, and illustrate the BPHZ treatment of the ghost number anomaly.

\section{Batalin-Vilkovisky Lagrangian quantisation}

In this section, we recall the vocabulary of the Batalin-Vilkovisky (BV) formulation of gauge theories [3], including the formulation of anomalies on a formal quantum level. More detailed reviews on the BV scheme, reflecting the point of view of the present authors, can be found in [12, 13, 14]. Other recommended reading on BV is [15].

\footnotetext{
${ }^{1}$ This book is also useful for the reader who needs more background material on renormalisation and symmetries in field theory than can be provided in this paper.
} 


\subsection{Basic concepts}

The BV scheme combines BRS quantisation [16] and its extension to open algebras [17] with the observation -originally due to Zinn-Justin (see e.g. chapter 19 of [18])- that it is convenient to introduce sources for the BRST transformation rules during the renormalization process. With every field $\Phi^{A}$ one associates an antifield $\Phi_{A}^{*}$ of opposite Grassmann parity antifields are canonically conjugated w.r.t. the antibracket defined by

$$
(F, G)=\frac{\overleftarrow{\delta} F}{\delta \Phi^{A}} \frac{\vec{\delta} G}{\delta \Phi_{A}^{*}}-\frac{\overleftarrow{\delta} F}{\delta \Phi_{A}^{*}} \frac{\vec{\delta} G}{\delta \Phi^{A}}
$$

The ghost number of a field and that of its antifield add up to -1 .

The BRST invariant, classical theory is then described by an extended action $S\left(\Phi, \Phi^{*}\right)$, formed by adding several pieces: the classical action, a term linear in the antifields $\Phi_{A}^{*}$ with coefficients $R^{A}$ equal to the BRS variation of the corresponding field $\Phi_{A}$ :

$$
S\left[\Phi, \Phi^{*}\right]=S_{0}[\Phi]+\Phi_{A}^{*} R^{A}[\Phi]+\ldots,
$$

and additional terms, at least quadratic in the antifields, which encode special features of the BRST algebra, e.g. its off-shell structure. This action is to be constructed in such a way that the classical master equation

$$
(S, S)=0,
$$

is satisfied. A solution of this equation (together with some suitable boundary conditions) always exists [19], whereas its local character, universally taken as one of the cornerstones of quantum field theory and therefore a desirable characteristic, can always be ensured under reasonable conditions on the gauge algebra generators [20] (see also [21] and references therein).

The antibracket, eq.(2.1), the extended action, eq.(2.2), and the classical master equation, eq.(2.3), play a central role in the theory. For example, the antibracket is used to perform canonical transformations of field variables and to implement gauge fixing (which becomes merely a change of basis). The extended action is used to define the BRS operation, and the master equation (2.3) guarantees then its on-shell nilpotence.

\subsection{The quantum master equation and anomalies}

In the quantum theory, equation (2.3) is modified. Indeed, from a purely formal point of view, gauge (or BRS) invariance at quantum level, with a given action $S$, is transcribed into the BV formalism as

$$
\frac{1}{2}(S, S)=i \hbar \Delta S
$$

with

$$
\Delta=\frac{\vec{\delta}}{\delta \Phi^{A}} \frac{\vec{\delta}}{\delta \Phi_{A}^{*}} .
$$

We call attention once again to the implicit notation (see the footnote on page 1 ) which implies that the sum over $A$ in (2.5) includes not only a summation over all (components of) the fields, but also an integration over the space-time point. This point is the same for both functional

\footnotetext{
${ }^{2} \Phi^{A}$ is the complete set of fields in the theory: gauge fields, matter fields, ghosts, antighosts, ...

${ }^{3}$ De Witt notation is assumed throughout the paper: the index $A$ is used to indicate the different fields, their components, and the space-time point on which they depend (unless it is explicitly displayed). In this way, a summation over $A$ includes not only discrete summations, but also integration over space-time. The derivatives are left and right functional derivatives
} 
derivatives. Accordingly, the expression eq.(2.5) is meaningless when acting on a local functional -in particular its use in eq.(2.4)! In fact, it could be viewed as an expression involving $\delta(0)$, and one may be tempted to neglect it for that reason, for example appealing to dimensional regularisation [3]. However, careful consideration leads one to conclude that in fact this term is potentially a source of anomalies [4]. In the Pauli-Villars scheme for example, to order $\hbar$, a definite expression can be associated to it. Then one has to decide whether this anomaly is genuine: if it can be avoided by adding an extra counterterm $\hbar M_{1}$, the classical action $S$ should be modified to a 'quantum action' $S_{q}=S+\hbar M_{1}+\ldots$ Without restrictions on $M_{1}$ this is in fact always possible (see, for instance, [3]), but here the locality requirement comes into play again: for local quantum field theory one will refuse to add anything but a local $M_{1}$. If one can not find a local $M_{1}$, the theory has a genuine anomaly, and (2.4) can not be satisfied. This reasoning can be extended to higher orders in $\hbar$. The formal quantum master equation is then

$$
\frac{1}{2}\left(S_{q}, S_{q}\right)=i \hbar \Delta S_{q}
$$

where $S_{q}$ is the previously mentioned quantum actionf, formally reducing to the classical action for $\hbar \rightarrow 0$.

In conclusion, within this framework it is the task of one's regularisation scheme to provide a well defined replacement for the purely formal equation (2.6).

\subsection{The Zinn-Justin equation}

In the quantum theory, the master equation can also be discussed at the level of the effective action $\Gamma\left[\varphi, \Phi^{*}\right]$, a functional of the classical fields $\varphi^{A}$ and of the sources for the BRST transformations $\Phi_{A}^{*}$. This functional is obtained from the generating functional of the connected diagrams by trading the sources $J_{A}$ for the classical fields $\varphi^{A}$ by a Legendre transformation, while keeping the BRST sources $\Phi_{A}^{*}$ fixed. Since $\Gamma$ incorporates all quantum corrections to the classical action into effective interactions, i.e. $\Gamma\left[\varphi, \Phi^{*}\right]=S\left(\varphi, \Phi^{*}\right)+\hbar \Gamma_{1}\left[\varphi, \Phi^{*}\right]+\ldots$, the quantum BRST structure is naturally described by the equation

$$
\frac{1}{2}(\Gamma, \Gamma)=\frac{\overleftarrow{\delta} \Gamma}{\delta \varphi^{A}} \frac{\vec{\delta} \Gamma}{\delta \Phi_{A}^{*}}=0
$$

of which the classical master equation can be considered to be the $\hbar=0$ limit.

Fulfilment of (2.7) ensures in fact gauge independence of the quantum theory. Instead, if the BRST symmetry is broken by loop corrections, i.e. the gauge symmetry is anomalous, equation (2.7) is replaced, according to Lam's quantum action principle 22, 23, by [24]

$$
\frac{1}{2}(\Gamma, \Gamma)=-i \hbar(\mathcal{A} \cdot \Gamma)
$$

On the right hand side, $(\mathcal{A} \cdot \Gamma)$ is the generating functional for 1PI diagrams with one insertion of a local operator $\mathcal{A}\left[\varphi, \Phi^{*}\right]$. The BV formalism gives an explicit albeit formal expression for it, related to the quantum master equation (2.6), namely $\mathcal{A}=\Delta S+(i / 2 \hbar)(S, S)$. In this way the operator $\mathcal{A}\left[\varphi, \Phi^{*}\right]$ makes explicit the breaking of the classical BRST structure due to quantum corrections and is interpreted as the BRST anomaly. The equation (2.7), in a slightly different guise without the sources corresponding to what are presently the antifields of the antighosts, was discovered by Zinn-Justin in a discussion on the renormalisation of non-abelian gauge theories. It is therefore called the Zinn-Justin equation and can be considered as a rewriting of the Veltman-Ward identity for the BRST symmetry.

\footnotetext{
${ }^{4}$ In the sequel, in the quantum context, we will use simply $S$ instead of $S_{q}$ to denote the full quantum action.
} 
A detailed discussion of the BV formalism and renormalisation using the effective action has been given in [25]. Further advocacy for the use of BV in the formulation of renormalisability can be found in 26 .

\section{$3 \quad$ BPHZ}

To put the quantum theory of the BV method on a more solid footing, one wants a proper quantum version of the basic equations. In particular, because of its central importance, we are looking for a regularised version of the quantum master equation eq.(2.6) as an operator equation which is valid to all orders in perturbation theory. In this section we first discuss some alternatives, and then give our reasons for using the setup of Bogoliubov, Parasiuk, Hepp and Zimmerman. We continue with a summary of the basics of that method, and recall the treatment of the chiral anomaly as a paradigm.

\subsection{Alternatives}

As was already pointed out, the quantum modifications in eq.(2.6) as compared to eq.(2.3), and in particular a non-zero $\Delta S$, signal the possible appearance of anomalies. In fact, $\Delta S$ is closely related to the Jacobian of the path integral measure under BRST transformations, with a potentially diverging determinant. Let us therefore consider the method of Fujikawa [27]. The BRST Jacobian determinant is a product of eigenvalues of some operator in an infinite dimensional space, and this is regularised by introducing a Gaussian damping, removing the contribution of the 'high-energy' modes. However, the method does not provide an unambiguous rule for choosing the regulator (the Gaussian damping), although the ad hoc conditions that are sometimes imposed to obtain a consistent anomaly can be replaced by a more systematic approach using Pauli-Villars methods [28]. Also, the extension of the method to higher loops is not straightforward.

In contrast with the philosophy of the Fujikawa method, which considers the relevant Jacobian determinant as the primary object to regularise, a more comprehensive point of view is that a regularisation scheme should take care of the divergences of the full theory (up to a certain order in perturbation theory). Most of the universally popular regularisation schemes are formulated as prescriptions to associate first a regularised, and in a second step a renormalised, finite expression to diverging loop diagrams. Let us then consider 'dimensional regularisation', which gives a method to replace the Feynman rules by an ' $n$-dimensional' generalisation, with $n$ complex. Let us note that in this scheme one does not have at one's disposal a Lagrangian corresponding to the regularised step. It is well known that, when symmetry properties of the Lagrangian one considers change with dimension, dimensional regularisation requires a delicate treatment to provide the same answers as other regularisation methods, notably when computing anomalies. In fact, in the initial BV-treatment dimensional regularisation was invoked to drop the anomalous terms. The lack of a Lagrangian seems to prohibit the formulation of the basic local BV equations in this scheme, although if one accepts the customary interplay of integer and complex dimensions one should refer to the treatment河 in [7].

We now turn to schemes that $d o$ have a straightforward Lagrangian interpretation. Originally due to Pauli and Villars, the simple device of subtracting from loops a compensating loop in which a particle with a very large mass circulates is the oldest among these. One writes down a Lagrangian for the original theory plus an additional piece where all terms involve one or more new massive (Pauli-Villars) fields. This is done in such a way that at this stage the theory is

\footnotetext{
${ }^{5}$ especially charming through its use of $d-4$ as an extra (global) variable of the theory, with a corresponding $\mathrm{BV}$-antivariable that plays a role in the treatment of the anomaly too!
} 
(one-loop) finite. Then one computes the effective action, and does the renormalisation. For a renormalisable theory this step absorbs the terms that would potentially diverge in the next step, where one lets all masses of the PV-fields tend to infinity. The contents of the original theory is defined to be what one obtains in this limit5. In this treatment, possible complications arise if the mass terms do not respect all symmetries of the original theory. Then the classical master equation is broken and one has to investigate carefully whether the breaking terms survive after taking the infinite mass limit. If they do, the theory may have an anomaly. This method goes together well with the BV formalism, and this way of computing the one loop anomaly is by now well-documented in the literature. It fits well because the method is based on a Lagrangian, just like the BV setup?.

The Pauli-Villars scheme only works well at one loop. One could imagine circumventing this restriction by using higher (covariant) derivatives in combination with the one loop PV scheme [29] However, constructing such covariant derivatives requires an understanding of the geometry behind the gauge symmetry. For some models, for instance the $W_{3}$ gravity model which we use as an example in this paper, such knowledge may be lacking.

Another resolution to the divergence problem, operationally very close to Schwinger's proper time method, consists in modifying the propagators to cut off the loop momentum integrals. The Lagrangian gauge invariant implementation of this idea is necessarily nonlocal. This method has been advocated [31] as a universal solution to the regularisation problems, and was applied to the present problem in [5]. The most striking virtue of this method, namely regularisation to all loop levels, brings also the computation of higher loop corrections within reach [6], and makes it into the primary alternative to the method applied in this paper. We refer to [5, 6] for a detailed exposition.

\subsection{Why BPHZ?}

In view of the coherence of the $\mathrm{BV}$ formalism, which rest very heavily on the use of a classical action functional, a properly defined quantum action functional is highly desirable, and preferably well defined functional integral expressions as well. When regularisation is formulated directly in terms of diagrams however, the path integral is just a shorthand notation for a collection of diagrams. This notation naturally suggests relations between correlation functions (=sets of diagrams), such as those obtained by functionally integrating by parts, the most familiar of these being the Schwinger-Dyson equations and Ward identities. Relations of this type are used heavily to set up the formal BV scheme, so we require that regularisation keeps them in manageable form.

Of all renormalisation methods, the BPHZ method is probably the most solidly founded one. In fact it is used as a touchstone (see for example [32]) for other methods to establish their respectability. It adresses head-on the key issue in the regularisation of quantum field theory, viz. the definition of products of local operators at the same space-time point. In BPHZ this is done via an explicit prescription of all their matrix elements. These matrix elements are defined in terms of Feynman diagrams. The diagrams are the same as when working formally, by which we mean disregarding the divergence problem. When applying the Feynman rules as they follow from the action, one obtains meaningless expressions. The BPHZ method is a definite prescription, order by order in perturbation theory, to supplement the Feynman rules so that one

\footnotetext{
${ }^{6}$ The $\delta(0)$ problem of eq.(2.4) does not arise, because the offending right hand side can be avoided completely 14] due to a cancellation between the original fields and the PV fields.

${ }^{7}$ We do not wish to distinguish here between Lagrangian and Hamiltonian methods, but rather set apart these methods from a purely diagrammatic approach. We will continue to use the term 'Lagrangian' to indicate both variants of action-based treatments.

${ }^{8}$ The prescriptions given in [29] are not quite correct, but have been amended in the recent literature, see [30].
} 
obtains meaningful expressions. Whereas the prescription is inspired by formal manipulations, 'subtracting infinite counterterms', in fact it makes no reference to such a dubious step. Let us remind the reader that the best-founded proofs of renormalizability of various theories rest on the application of this method. Let us also remind the reader that its prescription is universal, in that it applies irrespective of the order in perturbation theory.

The big asset of the BPHZ scheme is that renormalized correlation functions with insertions of arbitrary composite operators can be defined in the same way and with the same ease as diagrams related to Green's functions of elementary fields. The technical tool that does the job is a so-called 'normal product' operator, that we discuss in the next subsection. The normal products uniquely determine the finite expression associated with every diagram. The relations between correlation functions containing composite operators that one formally derives from path integrals, all have counterparts as relations between normal ordered products, and therefore as relations between renormalized correlation functions. Thus while the BPHZ method is not exclusively based on an action functional, it does provide well-defined quantum operators that correspond to classical functionals through the normal product concept, and it does provide counterparts to the classical equations obeyed by these functionals. The BPHZ scheme is therefore well-suited to formulate the basic local equations of the BV formalism.

\subsection{Basics of BPHZ renormalization}

Elaborating on the work of Bogoliubov, Parasiuk and Hepp [33], Zimmerman [34] set up a renormalization scheme in which renormalized Green functions with composite operator insertions are obtained in essentially the same way as renormalized Green functions of elementary fields. The method?, which bypasses the regularization step, determines the renormalized counterpart $I_{R}(p)$ of a divergent loop integral $I(p)$ associated with a specific one particle irreducible Green function by subtracting from its integrand the first few terms in the Taylor series around vanishing external momenta $p$, i.e.

$$
I(p)=\int \mathrm{d} k \mathcal{I}(p, k) \longrightarrow I_{R}(p)=\int \mathrm{d} k\left(1-t_{p}^{\delta}\right) \mathcal{I}(p, k) .
$$

The minimal value of $\delta$ is the superficial degree of divergence of the diagram (if the diagram is not finite by power counting). One-loop diagrams can unambiguosly be treated with eq.(3.1), but multiloop diagrams with overlapping divergences require the use of the forest formula 34] to determine the Taylor series to be subtracted (see section 6 for an explicit example).

In fact, the BPHZ renormalization scheme allows to define for any local composite operator $\mathcal{O}(x)$ a sequence of normal ordered composite operators

$$
\mathrm{N}_{a}[\mathcal{O}(x)]
$$

with $a$ an integer greater than or equal to the canonical dimension of the operator $\mathcal{O}(x)$ (counting 1 for every derivative, etc. ). A normal product is called minimally subtracted (resp. oversubtracted) when $a$ is equal to (resp. greater than) its canonical dimension. For a generic connected Green function of free field normal products $\mathrm{N}_{a_{i}}\left[\mathcal{O}_{i}\left(x_{i}\right)\right]$

$$
\left\langle\prod_{i} \mathrm{~N}_{a_{i}}\left[\mathcal{O}_{i}\left(x_{i}\right)\right]\right\rangle_{c},
$$

the order $\delta$ of the Taylor series in Zimmerman's prescription (3.1) is given in terms of the degrees $a_{i}$ as

$$
\delta=n+\sum_{i}\left(a_{i}-n\right)-\sum_{k} d_{k},
$$

\footnotetext{
${ }^{9}$ Useful general references, containing a more detailed exposition are [2, 11].
} 
where $n$ is the space-time dimension and the last term subtracts all the canonical dimensions $d_{k}$ of external lines.

The use of oversubtracted operators, while seemingly superfluous, is in fact one of the virtues of the BPHZ method and a cornerstone in its use for anomaly computations. Moreover, it is useful to introduce not one but a variety of oversubtracted normal products [22]. Indeed, the degree of oversubtraction of an operator can be propagated to subdiagramst ways, the only constraint being that the degree of oversubtraction of a subdiagram should be at least as large as the degree of oversubtraction of its subdiagrams. When subdiagrams are always maximally oversubtracted one speaks of isotropic normal products (all lines are treated in the same way), otherwise of anisotropic normal products. We do not need the most general anisotropic ones, but only the Gomes-Lowenstein [35] (GL) version,

$$
\mathrm{N}_{d_{1}+d_{2}+\alpha}\left[\left\{\mathcal{P}_{1}\right\} \mathcal{P}_{2}\right]
$$

for which the rule that determines the subtraction degrees $\delta_{\gamma}$ of subdiagrams $\gamma$ is modified to

$$
\delta_{\gamma}=n-\sum_{k} d_{k}+\sum_{i \neq i_{0}}\left(a_{i}-n\right)+ \begin{cases}d_{1}+d_{2}-n, & \text { if } i_{0} \in \gamma \text { and } L\left(\mathcal{P}_{2}\right) \subseteq E(\gamma) \\ d_{1}+d_{2}+\alpha-n, & \text { if } i_{0} \in \gamma \text { and } L\left(\mathcal{P}_{2}\right) \nsubseteq E(\gamma) \\ 0, & \text { if } i_{0} \notin \gamma\end{cases}
$$

where $i_{0}$ is the normal product vertex under consideration; $d_{l}$ the canonical dimension of the operator $\mathcal{P}_{l} ; L\left(\mathcal{P}_{2}\right)$, the set of lines associated with $\mathcal{P}_{2}$ and $E(\gamma)$ the set of external lines of the subdiagram $\gamma$. Note that this rule implies that if $\mathcal{P}_{1}$ is linear in the fields then there is no distinction between the isotropic and the anisotropic products.

Let us conclude this summary by indicating two fundamental and useful properties of the normal ordered composite operators. The first of these, the BPHZ renormalized version of the Schwinger-Dyson equations [35], is [ా]

$$
\begin{gathered}
i \hbar\left\langle\mathrm{N}_{a}[\mathcal{O}(x)] \frac{\vec{\delta}}{\delta \Phi^{B}(x)}(-1)^{\epsilon_{B}}\left(\Phi^{A_{1}}\left(x_{1}\right) \ldots \Phi^{A_{m}}\left(x_{m}\right)\right)\right\rangle= \\
\left\langle\left(\mathrm{N}_{a+n-d_{B}}\left[\mathcal{O}(x) \frac{\overleftarrow{\delta} S_{0}}{\delta \Phi^{B}(x)}+\{\mathcal{O}(x)\} \frac{\overleftarrow{\delta} \tilde{S}_{\mathrm{I}}}{\delta \Phi^{B}(x)}\right]\right) \Phi^{A_{1}}\left(x_{1}\right) \ldots \Phi^{A_{m}}\left(x_{m}\right)\right\rangle
\end{gathered}
$$

where $d_{B}$ is the engineering dimension of the field $\Phi^{B}(x) ; S_{0}$, the massless free part of the action

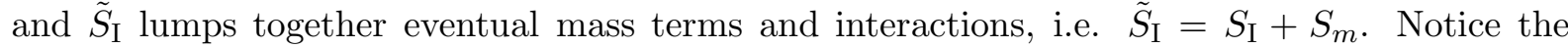
anisotropic normal ordering in the second term on the rhs.

The second property, the so-called Zimmerman identity, establishes linear relations among different normal products of a given monomial. Different formulations may be given. A first version relates normal products with different subtraction degrees $\alpha_{1}>\alpha_{2}$ to all operators $\mathcal{O}_{i}(x)$ having the same quantum numbers and with canonical dimensions $\leq \alpha_{1}$

$$
\mathrm{N}_{\alpha_{1}}[\mathcal{O}(x)]-\mathrm{N}_{\alpha_{2}}[\mathcal{O}(x)]=\sum_{i} r_{i} \mathrm{~N}_{\alpha_{1}}\left[\mathcal{O}_{i}(x)\right]
$$

\footnotetext{
${ }^{10}$ We always refer to proper subdiagrams, i.e. subdiagrams that are themselves $1 \mathrm{PI}$.

${ }^{11}$ The Taylor series operator acts on all momentum factors produced by the $\mathcal{O}_{i}$, but not on the propagators of the lines that are external to the subdiagram $\gamma$.

${ }^{12}$ Here and in the following, to avoid unnecessarily heavy notations, we act as if there are no dimensionful parameters in the model, and all operators in the action are minimally subtracted $\mathrm{N}_{n}[\cdot]$-products. It is rather trivial to overcome this restriction.
} 
where the coefficients $r_{i}$ vanish at lowest order. Our approach in fact uses a consequence of (3.6), relating oversubtracted operators to their minimally subtracted counterpart

$$
\mathrm{N}_{a+\alpha}[\mathcal{O}(x)]-\mathrm{N}_{a}[\mathcal{O}(x)]=\sum_{i} r_{i} \mathrm{~N}_{a_{i}}\left[\mathcal{O}_{i}(x)\right]
$$

where now all the operators on the rhs are minimally subtracted. A second, extended version of the Zimmerman identity further relates anisotropic and isotropic normal products with the same substraction degree

$$
\mathrm{N}_{d_{1}+d_{2}+\alpha}\left[\left\{\mathcal{P}_{1}\right\} \mathcal{P}_{2}\right]-\mathrm{N}_{d_{1}+d_{2}+\alpha}\left[\mathcal{P}_{1} \mathcal{P}_{2}\right]=\sum_{i} r_{i} \mathrm{~N}_{a_{i}+d_{2}}\left[\mathcal{O}_{i}(x) \mathcal{P}_{2}\right]
$$

where the same restrictions for $a_{i}$ and $r_{i}$ apply also in this case. In the next section, the two relations (3.5) and (3.7) will be used extensively for the implementation of BPHZ ideas into the BV framework.

\subsection{Anomalies and the massless case}

The exposure of the chiral anomaly in the present framework is an application of the BPHZ method that dates back some 25 years. We shall not repeat this calculation here, since a short account of it can be found in 9], but just sketch the main ideas and stress that such recipe works in general for determining the anomaly in a global, continuous symmetry.

The central equation, which computes the divergence of (matrix elements of) the axial vector current in Q.E.D. ([9], eqs. (1.7) and (1.8)) is

$$
\begin{gathered}
\partial_{\mu}\left\langle\mathrm{N}_{3}\left[\bar{\psi} \gamma^{\mu} \gamma^{5} \psi\right](x) X\right\rangle= \\
-\left\langle\left[\left(\gamma_{5} \psi(x)\right) \frac{\delta}{\delta \psi(x)}+\left(\bar{\psi}(x) \gamma_{5}\right) \frac{\delta}{\delta \bar{\psi}(x)}\right] X\right\rangle+2 i m\left\langle\mathrm{~N}_{4}\left[\bar{\psi} \gamma^{5} \psi\right](x) X\right\rangle
\end{gathered}
$$

which is an application of eq. (3.5). Then the oversubtracted operator $\mathrm{N}_{4}\left[\bar{\psi} \gamma^{5} \psi\right]$ is rewritten in terms of minimally subtracted ones by using Zimmerman's identity (3.7) as

$$
\mathrm{N}_{4}\left[\bar{\psi} \gamma^{5} \psi\right]=\mathrm{N}_{3}\left[\bar{\psi} \gamma^{5} \psi\right]+r \mathrm{~N}_{4}\left[F_{\mu \nu} \tilde{F}^{\mu \nu}\right]+s \mathrm{~N}_{4}\left[\partial_{\mu}\left(\bar{\psi} \gamma^{\mu} \gamma^{5} \psi\right)\right]
$$

An important feature of the treatment is that, for massless fermions, a mass has to be introduced to perform the calculation. The reason is that the BPHZ method introduces an apparent infrared divergence in the subtraction terms, in spite of the fact that no physical infrared problems are associated with the vanishing of the electron mass. It comes up when one tries to calculate the matrix elements of the oversubtracted operator $\mathrm{N}_{4}\left[\bar{\psi} \gamma^{5} \psi\right]$ : they diverge in the massless limit. Ultimately, this is why one is forced to reduce the oversubtracted to an ordinary normal product: the latter has matrix elements that stay finite when the mass goes to zero (if there is no physical infrared problem). The upshot in the present computation is that, in eq. (3.9), when $m \rightarrow 0$, one picks up the terms from eq.(3.10) that go as $m^{-2}$. This is the origin of the axial anomaly. For the actual computation we refer to the quoted literature for the axial anomaly, and for other examples to sections 5.3 and 6.3 and appendix $\mathrm{Q}$ of this paper.

\section{Anomalies in the BV-BPHZ renormalized framework}

The properties of the normal ordered products (3.5), (3.6) have been known to provide a natural framework for the investigation of anomalies in global symmetries for a long time. Furthermore, they were extensively used in the original presentation of the BRS quantization 
scheme [16]. We will carry these ideas one step further by implementing BPHZ ideas in the $\mathrm{BV}$ framework, in order to formulate its basic local equations -essentially the quantum master equation- on the level of renormalised perturbation theory and to derive a proper method to compute higher loop anomalies. For treatments on the level of the effective action, which have been limited to one-loop anomalies, we refer to [11] and references therein.

Roughly speaking, this program is realized by substituting formal products of fields by suitable Zimmerman normal products. In the assignment of dimensions $d^{A}, d_{A}^{*}$ to the fields $\Phi^{A}$ and antifields $\Phi_{A}^{*}$, there is a certain degree of arbitrariness, although they are largely fixed by the classical free Lagrangian. This arbitrariness is fixed in a way that is convenient for the BV framework by demanding that the BRST operator preserves canonical dimensions [3]. The following relations then hold

$$
d\left[\frac{\delta S}{\delta \Phi^{A}}\right]=n-d^{A}, \quad d\left[\frac{\delta S}{\delta \Phi_{A}^{*}}\right]=d^{A}=n-d_{A}^{*},
$$

such that the dimensions of a field and of its corresponding antifield add up to the space-time dimension $n$. As a consequence, normal products involving BRST sources obey, for example

$$
\mathrm{N}_{a}\left[\Phi_{A}^{*} R^{A}\right]=\Phi_{A}^{*} \mathrm{~N}_{a-n+d_{A}}\left[R^{A}\right]
$$

An essential ingredient to deal with massless theories within our program consists in the introduction of a small mass $m$ for the massless fields. As was pointed out in sec. 3.4, this is necessary to prevent the appearance of spurious IR divergences when performing the prescribed subtractions at vanishing external momenta 4 . In these cases, one therefore replaces the original massless action $S$ by a massive action $\tilde{S}$

$$
\tilde{S}\left(\Phi, \Phi^{*}\right)=S\left(\Phi, \Phi^{*}\right)+\frac{m^{2}}{2} S_{m}(\Phi)=S+\frac{m^{2}}{2} \Phi^{A} T_{A B} \Phi^{B},
$$

supplemented with the rule that the limit $m \rightarrow 0$ is taken at the very end of the renormalized computations. Also, $d^{A}+d^{B}+d\left(T_{A B}\right)=n-2$.

We will now formulate the basic equations governing the BV method at the renormalised perturbative level. As already pointed out in the introduction, in the BV scheme the quantum BRST structure and its possible violations are naturally described by means of the BRSTVeltman-Ward identity (2.8) for the effective action $\Gamma$. To write down the corresponding local equation we start from the generating functional of all connected Green functions $W(J)$, of which $\Gamma$ is the Legendre transform, $\Gamma\left(\Phi, \Phi^{*}\right)=W\left(J, \Phi^{*}\right)-J \Phi$, where it is understood that to obtain the left hand side one should solve the equation $\Phi=\frac{\delta W}{\delta J}$ for $J$ as a function of $\Phi$ and $\Phi^{*}$. By inverting the Legendre transform, one then also has that $\frac{\delta \Gamma}{\delta \Phi}=-J$. The left hand side of equation (2.8) is then equal to

$$
-\int \mathrm{d}^{n} x J_{A}(x) \frac{\vec{\delta} \Gamma}{\delta \Phi_{A}^{*}(x)} .
$$

\footnotetext{
${ }^{13}$ If one follows the traditional assignment, of 9] for instance, the usual Yang-Mills ghosts and antighosts would both have (scaling) dimension 1, whereas we assign dimension 0 to $c$ and 2 to $b$, as in ref. 11. Also, antifields would have 0 dimension (being external fields), whereas our antifields do not. The BRST operator increases that (traditional) scaling dimension by 1 unit. We will adopt conventions matching those of conformal field theory (see table 1 on page 16 and table 1 on page 24). The difference is only one of bookkeeping.

${ }^{14}$ Subtraction at non-zero momentum is feasable, but unnecessarily complicated regarding counterterms and Lorentz invariance.
} 
A general property of Legendre transformations is that the derivatives of $\Gamma$ and $W$ with respect to any parameter, $\Phi^{*}$ for example, are equal:

$$
\frac{\vec{\delta} \Gamma\left(\Phi, \Phi^{*}\right)}{\delta \Phi_{A}^{*}(x)}=\frac{\vec{\delta} W\left(J, \Phi^{*}\right)}{\delta \Phi_{A}^{*}(x)}
$$

where on the l.h.s $\Phi$ is kept constant in taking the derivative, and on the r.h.s. $J$ is kept constant, and it is understood that after taking the derivatives the functional relation between $J$ and $\Phi$ is imposed. Since $W$ is nothing but the generating functional of all the connected Green functions, a (formal) power series in the source $J$, its derivative with respect to the parameters $\Phi^{*}$ is the generating functional of all connected Green functions with one extra insertion of the operator $\frac{\vec{\delta}_{S}}{\delta \Phi_{A}^{*}(x)}$. Suppressing the $\Phi^{*}$ dependence in the notation, we will denote the resulting generating functional with the insertion of $X$ by $\langle X\rangle_{c, J}$, so that, for example, $W\left(J, \Phi^{*}\right)=\langle\mathbf{1}\rangle_{c, J}$. This local functional of the fields (and possibly of antifields as well) that one has to insert is not just the classical functional (which would be ill-defined), but one of the normal products that correspond to this classical functional, which have a definite meaning in the BPHZ scheme. The particular normal product one takes is an integral part of the definition of the quantum action functional one starts from. We take the interaction terms in $S$, in particular the antifield terms, to be minimally subtracted. Other choices differ in terms of order $\hbar$ or higher, see (3.7). One may wish to include in the action more general terms of higher order in $\hbar$. This would be the case when loop anomalies are to be canceled by finite non-invariant counterterms (usually called $M_{1}, M_{2}, \ldots$ in quantum $\mathrm{BV}$ formalism). We will continue to denote the full quantum action, including these terms if they are introduced, by the symbol $S$. (If some of these terms contain antifields, they are to be viewed as quantum modifications of the BRST transformation laws.) The normal ordering to be used for the lhs is then, according to (4.1), $\mathrm{N}_{d^{A}}[\cdot]$. The equation (2.8) is then completely equivalent with

$$
\int \mathrm{d}^{n} x J_{A}(x)\left\langle\mathrm{N}_{d^{A}}\left[\frac{\vec{\delta} S}{\delta \Phi_{A}^{*}(x)}\right]\right\rangle_{c, J}=i \hbar \int \mathrm{d}^{n} x\left\langle\mathrm{~N}_{n}[\mathcal{A}(x)]\right\rangle_{c, J}
$$

It should be emphasized that this equation is an exact one, involving on the l.h.s. only quantities which are well-defined in renormalised perturbation theory, although rather empty until one specifies the operator $\mathrm{N}_{n}[\mathcal{A}(x)]$ on the r.h.s.: in fact one may take it as the definition of $\mathcal{A}$. If one treats the equation formally, ignoring the normal product rules, for example using the heuristic path integral formulas, then, as already mentioned in section 2.3, it is rather easy to derive the general formal expression $\mathcal{A}=\Delta S+\frac{i}{2 \hbar}(S, S)$, for which there is of course no place in the BPHZ scheme. Reverting to a more proper treatment, there is still a general statement that can be made: a celebrated result of general BPHZ renormalisation theory, namely Lam's theorem [22, 23] (also called the quantum action principle) guarantees that the anomaly insertion $\mathcal{A}(x)$ in (2.8) is the integral of a local composite operator, whose canonical dimension is $n$ in our conventions ${ }^{15}$, without specifying its explicit form. We will not need to rely on this general theorem here, since we will now deduce an explicit formula for $\mathcal{A}$ that shows this locality explicitly.

Expanding (4.6) in the sources $J_{A}$ one obtains

$$
-\int \mathrm{d}^{n} x\left\langle\left(\mathrm{~N}_{d^{A}}\left[\frac{\vec{\delta} S}{\delta \Phi_{A}^{*}(x)}\right] \frac{\vec{\delta}}{\delta \Phi^{A}(x)}(-1)^{\epsilon_{A}}\right) \Phi^{A_{1}}\left(x_{1}\right) \ldots \Phi^{A_{m}}\left(x_{m}\right)\right\rangle_{c}
$$

\footnotetext{
${ }^{15}$ The well-known $\gamma_{5}$ anomaly in 4 dimensions, including the factor $c$, would have canonical dimension 5 if we had adopted the counting with the usual scaling dimensions.
} 


$$
=\int \mathrm{d}^{n} x\left\langle\mathrm{~N}_{n}[\mathcal{A}(x)] \Phi^{A_{1}}\left(x_{1}\right) \ldots \Phi^{A_{m}}\left(x_{m}\right)\right\rangle_{c}
$$

These equations are nothing but the typical anomalous Ward identities for the usual BV-BRST transformation $\delta$ since, for antifield independent functionals, one has classically that

$$
\delta F[\Phi]=\int \mathrm{d}^{n} x\left\{\frac{\vec{\delta} S}{\delta \Phi_{A}^{*}(x)} \frac{\vec{\delta}}{\delta \Phi^{A}(x)}(-1)^{\epsilon_{A}}\right\} F[\Phi]=(F, S) .
$$

To obtain from eq.(4.7) an equation in terms of local quantum operators (instead of correlation functions), we apply the BPHZ expression of the Schwinger-Dyson (SD) equation, in the form given by Gomez and Löwenstein, $(3.5)$, to its lhs, for the operators $\mathcal{O}(x)=\frac{\vec{\delta}_{S}}{\delta \Phi_{A}^{*}(x)}$. This leads to the following operator identity in terms of (possibly oversubtracted) anisotropic normal products

$$
\begin{gathered}
-i \hbar \int \mathrm{d}^{n} x \mathrm{~N}_{n}[\mathcal{A}(x)]= \\
\int \mathrm{d}^{n} x\left(\mathrm{~N}_{n}\left[\frac{\overleftarrow{\delta} S_{0}}{\delta \Phi^{A}(x)} \frac{\vec{\delta} S}{\delta \Phi_{A}^{*}(x)}\right]+\mathrm{N}_{n}\left[\frac{\overleftarrow{\delta}\left(S_{m}+S_{I}\right)}{\delta \Phi^{A}(x)}\left\{\frac{\vec{\delta} S}{\delta \Phi_{A}^{*}(x)}\right\}\right]\right)
\end{gathered}
$$

Assuming the validity of the classical master equation (2.3) for $S=S_{0}+S_{I}$, the functionals inside the normal ordering can be simplified, resulting in

$$
\begin{gathered}
-i \hbar \int \mathrm{d}^{n} x \mathrm{~N}_{n}[\mathcal{A}(x)]= \\
\int \mathrm{d}^{n} x\left(\mathrm{~N}_{n}\left[\frac{\overleftarrow{\delta} S_{I}}{\delta \Phi^{A}(x)}\left\{\frac{\vec{\delta} S}{\delta \Phi_{A}^{*}(x)}\right\}\right]-\mathrm{N}_{n}\left[\frac{\overleftarrow{\delta} S_{I}}{\delta \Phi^{A}(x)} \frac{\vec{\delta} S}{\delta \Phi_{A}^{*}(x)}\right]\right) \\
+\int \mathrm{d}^{n} x \mathrm{~N}_{n}\left[\frac{\overleftarrow{\delta} S_{m}}{\delta \Phi^{A}(x)}\left\{\frac{\vec{\delta} S}{\delta \Phi_{A}^{*}(x)}\right\}\right]
\end{gathered}
$$

This is the promised explicit local expression for the BRST anomaly in the BPHZ framework. We now comment on the different contributions in (4.9). The first contribution, potentially present in any interacting theory, comes from the difference between the anisotropic and the isotropic normal products of the BRST variation of the interaction term $S_{I}$, as a straightforward application of the generalized Zimmerman identity (3.8) shows. These contributions vanish however for minimally subtracted ${ }^{\mathrm{W}}$ BRST variations of the interaction, since then the GL anisotropic normal products (3.3, 3.4) and the isotropic ones exactly coincide. The second contribution to the anomaly originates in the explicit breaking of the BRST (or gauge) symmetry by the IR regulating mass term $S_{m}$ in 4.3

$$
\lim _{m \rightarrow 0} m^{2} \int \mathrm{d}^{n} x \mathrm{~N}_{n}\left[\Phi^{A} T_{A B}\left\{\frac{\vec{\delta} S}{\delta \Phi_{B}^{*}}\right\}(x)\right] .
$$

The massless limit may result in a non-zero contribution, as can be seen through a generalization of the argument presented in sec. 3.4. The composite operator in (4.10) has dimension $n-2$

\footnotetext{
${ }^{16}$ In [35] it is indicated that in nonlinear $\sigma$-models one may prefer non-minimal interaction terms. Therefore, these terms can not be dropped in general, although in the examples of this paper they could. See also footnote 12 on page 9
} 
(or $n-1$ for Fermi fields, for which the typical mass term in (4.3) would be proportional to $m$ instead of $\mathrm{m}^{2}$ ). Due to the normal ordering degree $n$, its insertion in correlation functions leads to oversubtracted integrals, so that taking the limit $m \rightarrow 0$ naively by simply discarding such contributions is incorrect. To compute this limit correctly requires a conversion to minimally subtracted operators first. We do this conversion in two steps. First we use the Zimmerman identity (3.8) to relate the anisotropic, oversubtracted normal product to its isotropic, oversubtracted counterpart, where the normal product corrections are expressed in terms of operators $\mathcal{M}_{k}(x)$ with canonical dimension $d_{k} \leq n$, i.e.

$$
\mathrm{N}_{n}\left[\Phi^{A} T_{A B}\left\{\frac{\vec{\delta} S}{\delta \Phi_{B}^{*}}\right\}(x)\right]=\mathrm{N}_{n}\left[\Phi^{A} T_{A B} \frac{\vec{\delta} S}{\delta \Phi_{B}^{*}}(x)\right]+\sum_{k} b_{k}^{\prime} \mathrm{N}_{d_{k}}\left[\mathcal{M}_{k}(x)\right] .
$$

In a second step, the isotropic, oversubtracted operator is expressed in terms of minimally subtracted normal products by means of the second Zimmerman identity (3.7). The net result of this process is then written as

$$
\mathrm{N}_{n}\left[\Phi^{A} T_{A B}\left\{\frac{\vec{\delta} S}{\delta \Phi_{B}^{*}}\right\}(x)\right]=\mathrm{N}_{n-2}\left[\Phi^{A} T_{A B} \frac{\vec{\delta} S}{\delta \Phi_{B}^{*}}(x)\right]+\sum_{k} b_{k} \mathrm{~N}_{d_{k}}\left[\mathcal{M}_{k}(x)\right],
$$

where the numerical coefficients $b_{k}$ are at least of first order both in coupling constants and in $\hbar$. After substitution of (4.11) in (4.10), the limit $m \rightarrow 0$ can be taken. If the theory has no infrared singularities the minimally subtracted normal products have finite matrix elements and consequently only those terms will contribute to eq.(4.10) that have coefficients $b_{k}$ of order $1 / \mathrm{m}^{2}$. The corresponding operators determine the second contribution to the anomaly in eq.(4.9). If there are infrared singularities, the effective action $\Gamma$ strictly speaking does not exist. A treatment of a Wilson-type effective action can certainly be developed, and a corresponding local operator treatment, and it seems reasonable to anticipate that, as long as the infrared divergences are merely logarithmic the treatment given above remains valid

To conclude our theoretical approach, we remark that the contribution to the anomaly generated by the IR regulating mass term is not uniquely fixed. Indeed, there may be more than one way to construct a mass term in (4.3). Different mass terms may behave differently under some symmetries, causing the anomaly to show up in different symmetries. A similar ambiguity is present in the context of the one-loop Pauli-Villars regularisation (see [28, 10], and [36] for an example how this can be exploited), but we have not investigated whether this correspondence is complete in all details.

In summary, the simple rule of substituting formal products of fields by suitable Zimmerman normal products has led us to an explicit expression, eq.(4.9), for the anomaly in the BV-BPHZ renormalized framework, which is theoretically quite interesting but not necessarily practical. In fact, it turns out that for the actual computation of the BRST anomaly insertion $\mathcal{A}$ in (2.8), eq.(4.7) is more convenient once an enumeration of possible monomials in $\mathcal{A}$ has been given. The next section will illustrate this by computing the conformal anomaly in chiral $W_{2}$ gravity using both alternatives. This will of course also serve as an illustration for the more abstract development of this section. In the section after that, we will go on and apply the method to the one and two-loop anomaly of $W_{3}$ gravity, mainly using the anomalous Ward identities (4.7), but the two-loop anomaly will also be derived from eq.(4.9).

\section{The anomaly of chiral $W_{2}$ gravity}




\subsection{The model}

Chiral $W_{2}$-gravity is a 2-dimensional model $(n=2)$ describing $D$ matter fields $\phi^{i}, i=$ $1, \ldots, D$, coupled to a gravitational gauge field through their energy-momentum tensor $T=$ $1 / 2\left(\partial \phi^{i}\right)\left(\partial \phi^{i}\right)$. A convenient, gauge-fixed extended action $[77$ for this model can be taken from 21]:

$$
\begin{aligned}
S=\int \mathrm{d}^{2} x \quad & \left\{\left[-\frac{1}{2}\left(\partial \phi^{i}\right)\left(\bar{\partial} \phi^{i}\right)+b(\bar{\partial} c)\right]\right. \\
& \left.+\phi_{i}^{*}\left[c\left(\partial \phi^{i}\right)\right]+b^{*}[-T+2 b(\partial c)+(\partial b) c]+c^{*}[(\partial c) c]\right\} \\
= & S_{0}+\int \mathrm{d}^{2} x \Phi_{A}^{*}(x) R^{A}(x) .
\end{aligned}
$$

In writing (5.1) the following conventions have been used:

$$
\partial=\partial_{+}, \quad \bar{\partial}=\partial_{-}, \quad x^{ \pm}=\frac{1}{\sqrt{2}}\left(x^{1} \pm x^{0}\right) .
$$

The fields $c$ and $b$ in (5.1) are respectively the ghost and antighost of the spin $j=2$ gauge symmetry, and $\left\{\phi_{i}^{*}, c^{*}, b^{*}\right\}$ are the antifields or sources for the BRST transformations for all fields. Remarkably, in this formulation interactions are completely contained in the antifield dependent terms, so that BRST sources can be regarded as coupling constants, in which perturbative expansions can be performed. In fact, the antifield $b^{*}$ appears in (5.1) as the source for (minus) the total energy-momentum tensor of matter and ghosts, viz.

$$
R^{b}=-\frac{1}{2}\left(\partial \phi^{i}\right)\left(\partial \phi^{i}\right)+2 b \partial c+(\partial b) c=-T-T_{\text {gh }} \equiv-T_{t} .
$$

Thus $b^{*}$ takes over the rôle of (minus) the gravitational gauge field.

We will work with the more general expression for the ghost energy momentum tensor

$$
T_{j}=-j b \partial c+(1-j)(\partial b) c,
$$

for a generic spin $(j, 1-j) b c$-ghost system. At the moment we need $j=2$; in the next section, for chiral $W_{3}$, we will have both spin $j=2$ and $\operatorname{spin} j=3$ ghost sectors.

For the assignment of dimensions, we follow common practice in conformal field theory. There are two conformal dimensions for each field, corresponding to the left and right Virasoro algebras. The total dimension $d$ is the sum of these, the spin $j$ is the difference. The relevant assignments are collected in table 1. For the antifields, we extended the assignments in accordance with eq.(4.1), and spin conservation. Note that the fields and the $\partial$ derivative have vanishing $d-j$, whereas antifields and the $\bar{\partial}$ derivative all have $d-j=2$. This will be useful in our analysis.

These dimensions do not correspond to the assignments used in section 3.3: a canonical assignment would attribute dimension $1 / 2$ to all ghost fields. The subtraction rules in eq.(3.2) and eq.(3.4) require modifications that reflect this. We quote the resulting modification only for the case of operator products that do not contain antifields (which should be extracted first using eq.(4.2)), both for $W_{2}$ and $W_{3}$ :

$$
\delta=n+\sum_{i}\left(a_{i}-n\right)+\sum_{k}^{\prime}\left(d_{k}-1\right),
$$

where the primed sum runs only over ghost external lines. This new rule extends to the anisotropic subtraction degree (3.4), in which $\sum_{k} d_{k}$ should be replaced by $\sum_{k}^{\prime}\left(-d_{k}+1\right)$ as well.

\footnotetext{
${ }^{17}$ We take this at the same time to be the quantum action, and as in the general treatment of section 4 the minimally subtracted normal products are understood. With this understanding, and including no modifications $M_{i}$ of higher order in $\hbar$, it will turn out that the anomaly comes out in its conventional form.
} 


\begin{tabular}{||c||c|c|c|c||}
\hline & $\operatorname{dim} d$ & $\operatorname{spin} j$ & gh. $\sharp$ & $d-j$ \\
\hline \hline$\phi$ & 0 & 0 & 0 & 0 \\
$c$ & -1 & -1 & 1 & 0 \\
$b$ & 2 & 2 & -1 & 0 \\
\hline$\phi^{*}$ & 2 & 0 & -1 & 2 \\
$c^{*}$ & 3 & 1 & -2 & 2 \\
$b^{*}$ & 0 & -2 & 0 & 2 \\
\hline$\partial$ & 1 & 1 & 0 & 0 \\
$\bar{\partial}$ & 1 & -1 & 0 & 2 \\
\hline
\end{tabular}

Table 1: Additive charges of fields and antifields in the $W_{2}$ model.

Finally, for the reason mentioned in section 3.4, we introduce a mass term for all propagating fields. For the matter fields, this is conveniently done by including in $S_{m}$ (4.3) the term

$$
-\frac{m^{2}}{2} \int d^{2} x \phi^{i}(x) \phi^{i}(x) .
$$

The free propagator for the massive matter fields then becomes

$$
\left\langle\phi^{i}(x) \phi^{j}(y)\right\rangle_{0} \equiv \Delta^{i j}(x-y)=\delta^{i j} \frac{i \hbar}{\partial \bar{\partial}-m^{2}} \delta(x-y),
$$

with the subscript 0 indicating from now on free correlation functions, that is, the ones evaluated in the zero-th order in the perturbative expansion in antifields. Introducing a mass term for the ghosts is slightly more subtle. The obvious choice, $-m \int d^{2} x b c$, has the inconvenience of breaking spin $(j)$ invariance. This can be amended by using the non-local mass term

$$
-m^{2} \int d^{2} x b(x) \frac{1}{\partial} c(x) .
$$

The introduction of this nonlocality requires some comments. The extra mass terms recall a similar feature in Pauli-Villars regularisation. There, extra fields are introduced to regularise loop integrals, and these extra fields are given very large masses at the end of the calculation. Anomalies are understood as remnants of the fact that these mass terms do not respect all symmetries that were present classically. For example, in [28] the ghost number anomaly was traced to a mass term with ghost number different from 0 . There are some important differences with the BPHZ method followed in this paper. The most important one is that now the masses are given to the original fields (there are no extra fields), and they tend to zero at the end, not infinity. Thus the nonlocality of the mass term does not pose any problem in the present framework, whereas in the PV scheme it would. The locality is preserved here by the subtraction method (a Taylor series in momenta), and the introduction of masses is a device needed when oversubtracted operators appear. The consistency of the setup can be verified by checking that, despite having ghost number zero, this mass term reproduces correctly the well-known ghost number anomaly. This is demonstrated in appendix $\mathbf{Q}$. In fact the peculiar non-locality is at the origin of the ghost number anomaly. From a perturbative point of view, the nonlocal character of (5.7) poses no problem either. With this choice, the free propagator for the massive ghost fields

$$
\begin{gathered}
\langle c(x) b(y)\rangle_{0}=\langle b(x) c(y)\rangle_{0}=-\langle b(y) c(x)\rangle_{0} \\
\equiv G(x-y)=\frac{i \hbar \partial_{x}}{\partial \bar{\partial}-m^{2}} \delta(x-y) .
\end{gathered}
$$


takes a form that is common for Fermi fields. We conclude that the ghost mass term (5.7), though non-local, is indeed appropriate for our purposes.

\subsection{The $W_{2}$ anomaly from the anomalous Ward identities}

Now everything is ready to start the computation of the anomaly by using the anomalous Ward identities (4.7). Its evaluation can be divided in two steps. First, one uses all possible information concerning symmetries and quantum numbers to determine the general form of the monomials that can build up the anomaly. The connected character of the involved Green functions together with the presence of the normal products severely restricts the form of the anomaly candidates, so that their field content is completely determined. A standard perturbative computation then determines the coefficients of such anomaly candidates.

Let us now implement this program in the present example. Since the BRST transformation $\delta$, eq.(4.8), preserves spin $(j)$ the anomaly is a spin zero object, $j[\mathcal{A}(x)]=0$. Combining this information with the canonical dimension, $d[\mathcal{A}(x)]=2$ in our conventions, one finds $(d-$ $j)[\mathcal{A}(x)]=2$. From Table 1 it follows that all possible terms in the anomaly contain either precisely one antifield or precisely one $\bar{\partial}$-derivative. Lam's theorem further ensures that the numerical coefficients of the candidate anomalies are at least of first order in both $\hbar$ and the coupling constants, the antifields $\Phi^{*}$. Thus the anomaly has no $\bar{\partial}$ dependent terms, and is of the form $\mathcal{A}(x)=\Phi_{A}^{*}(x) F^{A}\left(\partial, \Phi^{B} ; x\right)$. This determines the antifield dependence of the anomaly completely.

Consider now the anomalous Ward identities (4.7). From the the above analysis, it is clear that all the relevant information about the functions $F^{A}(x)$-up to now, still arbitrary functions of $\partial$ and all fields- is contained in the loop contributions to the linear term in the antifield expansion of the lhs of (4.7), namely

$$
\begin{gathered}
\int \mathrm{d}^{2} y\left\langle\mathrm{~N}_{d^{A}}\left[R^{A}(x)\right]\left(\mathrm{N}_{d^{B}}\left[R^{B}(y)\right] \frac{\vec{\delta}}{\delta \Phi^{B}(y)}(-1)^{\epsilon_{B}}\right) \Phi^{A_{1}}\left(x_{1}\right) \ldots \Phi^{A_{m}}\left(x_{m}\right)\right\rangle_{c, 0} \\
=i \hbar\left\langle\mathrm{N}_{d^{A}}\left[F^{A}(x)\right] \Phi^{A_{1}}\left(x_{1}\right) \ldots \Phi^{A_{m}}\left(x_{m}\right)\right\rangle_{c, 0},
\end{gathered}
$$

where relation (4.2) was used. The form of the functions $F^{A}(x)$ can now be completely determined as follows. On the one hand, the normal ordered character of the insertions in (5.9) and the connectedness of the Green functions indicate that "proper" loops (i.e. no tadpoles, which are zero due to the subtractions) can only be generated by making at least two contractions

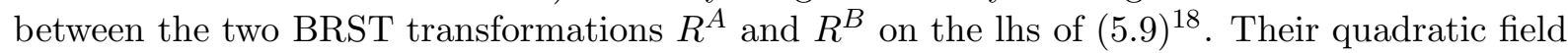
dependence restricts moreover the maximum number of contractions to be precisely two and leads thus to the conclusion that only one-loop anomalies can appear. On the other hand, from inspection of the free propagators (5.6), (5.8), it follows that these double contractions are only possible when both $A$ and $B$ refer to the antighost $b$. These contractions made, no fields are left to contract with, such that the "test product" of fields $\Phi^{A_{1}} \ldots \Phi^{A_{m}}$ must be equal to $b(z)$ in order to get a non-vanishing lhs. The lhs of (5.9) becomes

$$
-\int \mathrm{d}^{2} z\left\langle\mathrm{~N}_{2}\left[R^{b}(x)\right] \mathrm{N}_{2}\left[R^{b}(z)\right] \frac{\vec{\delta}}{\delta b(z)} b(y)\right\rangle_{c, 0}=-\left\langle\mathrm{N}_{2}\left[T_{t}(x)\right] \mathrm{N}_{2}\left[T_{t}(y)\right]\right\rangle_{c, 0},
$$

since $R^{b}=-T-T_{\text {gh }} \equiv-T_{t}$, see eq. (5.2).

The correlation function on the rhs of (5.9), of the form $\left\langle\mathrm{N}_{2}[F(x)] b(z)\right\rangle_{c, 0}$ with the above test product, is non-vanishing if and only if $F(x)$ contains precisely one $c$-ghost, since the presence of

\footnotetext{
${ }^{18} \mathrm{As}$ a consequence linear transformations do not contribute.
} 


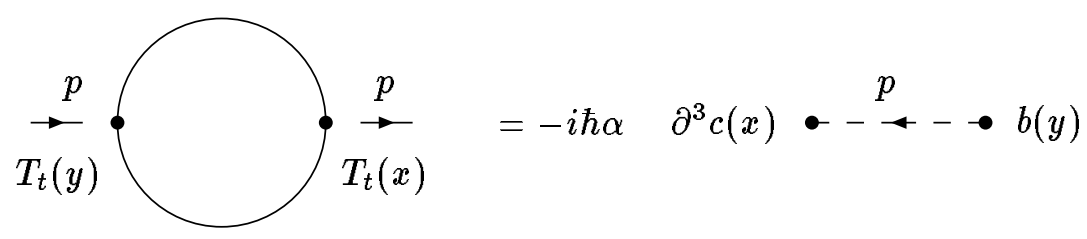

Figure 1: The one-loop anomaly diagram for $W_{2}$.

other fields in $F(x)$ would lead to extra tadpoles. Spin conservation implies $\mathcal{A}(x)=\alpha b^{*} F(x)=$ $\alpha b^{*} \partial^{3} c$, so that (5.9) becomes

$$
\left\langle\mathrm{N}_{2}\left[T_{t}(x)\right] \mathrm{N}_{2}\left[T_{t}(y)\right]\right\rangle_{c, 0}=-i \hbar \alpha\left\langle\mathrm{N}_{2}\left[\partial^{3} c(x)\right] b(y)\right\rangle_{c, 0}
$$

This is an explicit equation for the anomaly coefficient $\alpha$, which corresponds diagramatically to Figure 1. The coefficient $\alpha$ is thus determined by double contractions of the total energymomentum tensor $T_{t}$ with itself. Let us stress that the lhs of (5.10) is completely determined in renormalized perturbation theory, owing to the normal orderings. The equation expresses in the BPHZ language the well-known fact that double contractions in the OPE of $T_{t}(x) T_{t}(y)$ generate the conformal anomaly.

The anomaly computation is rounded off with the determination of the actual value of the coefficient $\alpha$ from eq. (5.10). On the lhs, the matter and ghost field contributions can be analyzed separately, since no contractions between $T$ and $T_{\text {gh }}$ are possible. The formal expression for the contribution of the matter sector is

$$
\begin{aligned}
\langle T(x) T(y)\rangle_{0} & =-\hbar^{2} \frac{D}{2}\left[\frac{\partial^{2}}{\partial \bar{\partial}-m^{2}} \cdot \delta(x-y)\right]^{2} \\
& =-\hbar^{2} \frac{D}{2} \int \frac{\mathrm{d}^{2} p}{(2 \pi)^{2}} e^{i p \cdot(x-y)} I_{22}(p, m)
\end{aligned}
$$

where $I_{22}(p, m)$ is a quadratically divergent integral (the explicit expression is in eq.(5.13) with $a=b=2$ ). The correct expression is similar, and arises by replacing the composite operators $T$ by their (minimally) normal ordered counterparts, $\mathrm{N}_{2}[T]$. The subtraction rule (5.4) states that the integrand $\mathcal{I}_{22}(q, p, m)$ in the quadratically divergent one-loop integral must be replaced by $\left(1-t_{p}^{2}\right) \mathcal{I}_{22}(q, p, m)$, confirming that minimally subtracted operators yield subtraction degrees precisely equal to the superficial degree of divergence. The correlation function of the normal ordered energy-momentum tensor for the matter sector is

$$
\left\langle\mathrm{N}_{2}[T(x)] \mathrm{N}_{2}[T(y)]\right\rangle_{c, 0}=-\hbar^{2} \frac{D}{2} \int \frac{\mathrm{d}^{2} p}{(2 \pi)^{2}} e^{i p \cdot(x-y)}\left(1-t_{p}^{2}\right) \cdot I_{22}(p, m),
$$

where we introduced the short hand notation

$$
I_{a b}^{R}(p, m) \equiv\left(1-t_{p}^{n}\right) \cdot I_{a b}(p, m) \equiv \int \frac{\mathrm{d}^{2} q}{(2 \pi)^{2}}\left(1-t_{p}^{n}\right) \mathcal{I}_{a b}(q, p, m),
$$

and $\mathcal{I}_{a b}(q, p, m)$ is the integrand of the formal integral

$$
I_{a b}(p, m)=\int \frac{\mathrm{d}^{2} q}{(2 \pi)^{2}} \frac{q^{a}(p-q)^{b}}{\left(q \bar{q}+m^{2}\right)\left[(p-q)(\bar{p}-\bar{q})+m^{2}\right]} \equiv \int \frac{\mathrm{d}^{2} q}{(2 \pi)^{2}} \mathcal{I}_{a b}(q, p, m),
$$


with $a, b \geq 1$. For general spin $j$ the formal expression

$$
\left\langle T_{j}(x) T_{j}(y)\right\rangle_{c, 0}=\hbar^{2} \int \frac{\mathrm{d}^{2} p}{(2 \pi)^{2}} e^{i p \cdot(x-y)}\left[\left(2 j^{2}-2 j+1\right) I_{22}(p, m)+\left(2 j^{2}-2 j\right) I_{31}(p, m)\right]
$$

is likewise converted to the well-defined

$$
\begin{gathered}
\left\langle\mathrm{N}_{2}\left[T_{j}(x)\right] \mathrm{N}_{2}\left[T_{j}(y)\right]\right\rangle_{c, 0}= \\
\hbar^{2} \int \frac{\mathrm{d}^{2} p}{(2 \pi)^{2}} e^{i p \cdot(x-y)}\left[\left(2 j^{2}-2 j+1\right)\left(1-t_{p}^{2}\right) \cdot I_{22}(p, m)+\left(2 j^{2}-2 j\right)\left(1-t_{p}^{2}\right) \cdot I_{31}(p, m)\right]
\end{gathered}
$$

The explicit computation of the BPHZ renormalized expression $I_{a b}^{R}(p)$ is straightforward, and details are relegated to appendix $\mathrm{A}$. We simply quote the final result in the limit $m \rightarrow 0$, namely $($ A.9)

$$
I_{a b}^{R}=-\frac{i}{2 \pi} \frac{p^{a+b-1}}{\bar{p}} B(a, b),
$$

with $B(a, b)$ the Euler Beta function. The renormalized correlation functions corresponding to (5.11), (5.14) are found to be

$$
\begin{aligned}
\left\langle\mathrm{N}_{2}[T(x)] \mathrm{N}_{2}[T(y)]\right\rangle_{c, 0} & =\frac{i \hbar^{2} D}{24 \pi} \int \frac{\mathrm{d}^{2} p}{(2 \pi)^{2}} e^{i p \cdot(x-y)} \frac{p^{3}}{\bar{p}} \\
\left\langle\mathrm{~N}_{2}\left[T_{j}(x)\right] \mathrm{N}_{2}\left[T_{j}(y)\right]\right\rangle_{c, 0} & =\frac{-i \hbar^{2}}{24 \pi}\left[2\left(6 j^{2}-6 j+1\right)\right] \int \frac{\mathrm{d}^{2} p}{(2 \pi)^{2}} e^{i p \cdot(x-y)} \frac{p^{3}}{\bar{p}}
\end{aligned}
$$

and the renormalized expression for the lhs of (5.10) is

$$
\left\langle\mathrm{N}_{2}\left[T_{t}(x)\right] \mathrm{N}_{2}\left[T_{t}(y)\right]\right\rangle_{c, 0}=\frac{i \hbar^{2}}{24 \pi}(D-26) \int \frac{d^{2} p}{(2 \pi)^{2}} e^{i p(x-y)} \frac{p^{3}}{\bar{p}} .
$$

After computing the rhs of $(\underline{5.10})$ in the momentum representation in the limit $m \rightarrow 0$

$$
-i \hbar \alpha\left\langle\partial^{3} c(x) b(y)\right\rangle_{c, 0}=-\hbar^{2} \alpha \int \frac{d^{2} p}{(2 \pi)^{2}} e^{i p \cdot(x-y)} \frac{p^{3}}{\bar{p}},
$$

a comparison of (5.17) and (5.18) gives

$$
\alpha=\frac{-i}{24 \pi}(D-26) .
$$

Combining this with the previous results about the functional form of the anomaly, one finally obtains

$$
\mathcal{A}=\frac{i(D-26)}{24 \pi} \int d^{2} x c \partial^{3} b^{*}
$$

a well-known result.

\subsection{The $W_{2}$ anomaly from Zimmerman identities}

In the previous subsection we showed the relation of the anomaly with the Ward identity for correlation functions. In this subsection we show its relation with the local operator equation (4.9). It may arise from two different sources: normal ordering corrections originating in the anisotropy of the variation of the interactions, and the symmetry breaking produced by the IR 
regulating mass term. If we take both the interaction terms and the transformations in (5.1) to be minimally subtracted normal products, only the second source contributes:

$$
\begin{gathered}
-i \hbar \int \mathrm{d}^{2} x \mathrm{~N}_{2}[\mathcal{A}(x)]= \\
\lim _{m^{2} \rightarrow 0} \int \mathrm{d}^{2} x\left(\mathrm{~N}_{2}\left[\frac{\overleftarrow{\delta} S_{m}}{\delta \Phi^{A}(x)}\left\{\frac{\vec{\delta} S}{\delta \Phi_{A}^{*}(x)}\right\}\right]-\mathrm{N}_{0}\left[\frac{\overleftarrow{\delta} S_{m}}{\delta \Phi^{A}(x)} \frac{\vec{\delta} S}{\delta \Phi_{A}^{*}(x)}\right]\right)
\end{gathered}
$$

We have inserted the term with the minimally subtracted operator, which is supposed to vanish in the limit $m \rightarrow 0$ anyway, to bring out the fact that classically this quantity is zero. The variation of the mass terms (5.5) and (5.7) reads explicitly

$$
\begin{gathered}
\mathrm{N}_{2}\left[\frac{\overleftarrow{\delta} S_{m}}{\delta \Phi^{A}(x)}\left\{\frac{\vec{\delta} S}{\delta \Phi_{A}^{*}(x)}\right\}\right]=-m^{2} \mathrm{~N}_{2}\left[\phi^{i}\left\{c \partial \phi^{i}\right\}(x)+\left(\frac{1}{\partial} c\right)\{T\}(x)\right] \\
+m^{2} \mathrm{~N}_{2}\left[\left(\frac{1}{\partial} b\right)\{(\partial c) c\}(x)+\left(\frac{1}{\partial} c\right)\{2 b \partial c+(\partial b) c\}(x)\right]
\end{gathered}
$$

A non-zero result for eq. $(5.20)$ arises from a difference in the subtractions. We first spell out the influence of the anisotropy on the various terms in eq.(5.21), and later turn to the influence of the oversubtractions. For the terms involving matter fields, when inserted in loop correlation functions 5 , the absence of interaction terms containing the product $b \phi^{i}$ forces the factor $\phi^{i}$ to be always connected to an internal line, whereas the factor $\left(\frac{1}{\partial} c\right)$, instead, is always connected to an external line. Both then produce matter loops only. As a consequence of the definition (3.4), one sees that the first of these anisotropic normal products behaves effectively as an isotropic, oversubtracted one, whereas the second reduces in fact to its isotropic, minimally subtracted version (and thus produces no contribution to the anomaly (5.20)). Similar conclusions can be drawn for the first of the pure ghost terms of eq.(5.21). Since the factor $\left(\frac{1}{\partial} b\right)$ is necessarily internal in a loop, the anisotropic normal ordering effectively behaves once again as an isotropic oversubtracted product. For the second pure ghost term, the net effect of the anisotropy is to first eliminate ghost loop contributions formed by considering the factor $\left(\frac{1}{\partial} c\right)$ as external, and to force the remaining ones to be isotropic, although oversubtracted.

Having disentangled the consequences of the anisotropy, the oversubtraction can now be taken into account. This is done through the Zimmerman identity (3.7)

$$
\mathrm{N}_{2}[\Theta(x)]-\mathrm{N}_{0}[\Theta(x)]=\sum_{k} \rho_{k} \mathrm{~N}_{d_{k}}\left[\mathcal{M}_{k}(x)\right]
$$

with $d\left[\mathcal{M}_{k}\right]=d_{k} \leq 2$, and with the composite operator $\Theta(x)$ given by

$$
\begin{gathered}
\Theta(x) \equiv \Theta_{\mathrm{m}}(x)+\Theta_{\mathrm{gh}}(x)= \\
-\phi^{i}\left\{c \partial \phi^{i}(x)\right\}+\left[\left(\frac{1}{\partial} b\right)\{(\partial c) c\}(x)+\left(\frac{1}{\partial} c\right)\{2 b \partial c+(\partial b) c\}(x)\right] .
\end{gathered}
$$

The coefficients are most easily determined by inserting both sides of $(5.22)$ into correlation functions with arbitrary products of fields. The insertion $\Theta(x)(5.23)$ has spin and dimension zero, which restricts the normal product corrections on the rhs of $(\overline{5.22})$ to be exactly of the form $\sum_{k} m^{-2} \tilde{\rho}_{k} \mathrm{~N}_{2}\left[\Phi_{A}^{*} F_{k}^{A}\right]$, with $\tilde{\rho}_{k}$ mass and coupling constant independent numerical coefficients.

\footnotetext{
${ }^{19}$ The only non-zero correlation functions that can be formed with (5.21) and elementary fields is $\langle\mathrm{N}[\mathcal{A}](x) b(y)\rangle_{0}$. See the previous subsection.
} 
The actual value of the coefficients $\tilde{\rho}_{k}$ and the field content of the objects $F_{k}^{A}$ is again contained in the loop contributions to the term linear in antifields, namely

$$
\begin{gathered}
\frac{i}{\hbar} \int d^{2} x \int d^{2} y\left\langle\mathrm{~N}_{2}\left[\Phi_{A}^{*}(x) R^{A}(x)\right]\left\{\mathrm{N}_{2}[\Theta(y)]-\mathrm{N}_{0}[\Theta(y)]\right\} \prod_{i} \Phi^{A_{i}}\left(x_{i}\right)\right\rangle_{c, 0}= \\
\sum_{k} \rho_{k} \int d^{2} x\left\langle\mathrm{~N}_{2}\left[\Phi_{A}^{*}(x) F_{k}^{A}(x)\right] \prod_{i} \Phi^{A_{i}}\left(x_{i}\right)\right\rangle_{c, 0} .
\end{gathered}
$$

Considering possible double contractions between $\Theta(y)$ and $R^{A}(x)=R^{b}(x)$, which always leave a factor $c(y)$ free, the test product of fields is again $b(z)$ and the operator on the rhs can only be $\Phi_{A}^{*}(x) F^{A}(x)=b^{*} \partial^{3} c(x)$. Differentiating w.r.t. the antifield $b^{*}$, (5.24) reduces to

$$
\begin{gathered}
-\frac{i}{\hbar} \int \mathrm{d}^{2} y\left\langle\mathrm{~N}_{2}\left[T_{t}(x)\right]\left\{\mathrm{N}_{2}[\Theta(y)]-\mathrm{N}_{0}[\Theta(y)]\right\} b(z)\right\rangle_{c, 0}= \\
\rho\left\langle\mathrm{N}_{2}\left[\partial^{3} c(x)\right] b(z)\right\rangle_{c, 0},
\end{gathered}
$$

which is again an equation for the numerical coefficient $\rho$ of the potential anomaly $\mathcal{A}=b^{*} \partial^{3} c$.

Let us now evaluate the contribution $\rho_{\mathrm{m}}$ of the matter sector -coming from double contractions between $\Theta_{\mathrm{m}}(y)(5.23)$ and $T(x)$ - to the coefficient $\rho$ in eq. (5.25). The BPHZ renormalized correlation function is

$$
\begin{aligned}
\Lambda_{a}(x-y) & \equiv \frac{i}{\hbar} \int \mathrm{d}^{2} z\left\langle\mathrm{~N}_{2}[T(x)] \mathrm{N}_{a}\left[\phi^{i}(z) c(z)\left(\partial \phi^{i}\right)(z)\right] b(y)\right\rangle_{c, 0} \\
& =\frac{\hbar^{2} D}{2} \int \frac{\mathrm{d}^{2} p}{(2 \pi)^{2}} e^{i p \cdot(x-y)} \frac{p^{2}}{p \bar{p}+m^{2}}\left(1-t_{p}^{a}\right) \cdot I_{11}(p, m) .
\end{aligned}
$$

The short hand notation (5.12) was used again. The property $t_{p}^{a+1} p=p t_{p}^{a}$ allows the commutation of the explicit factor $p$ coming from the $\partial c$ through the Taylor operator $t_{p}^{a+1}$, that is assigned by eq. (5.4) to the 1PI part $p I_{11}(p, m)$ of the above integral. For $a=0$ the new 1PI integral is seen to be minimally subtracted -indeed, $I_{11}(p, m)$ is logarithmically divergent (cf. appendix A) - while for $a=2$ it is oversubtracted. We are interested in the difference, see eq.(5.25), i.e. in the subtraction terms themselves, of zero and second order in $p$ :

$$
\Lambda_{2}(x-y)-\Lambda_{0}(x-y)=\frac{\hbar^{2} D}{2} \int \frac{\mathrm{d}^{2} p}{(2 \pi)^{2}} e^{i p \cdot(x-y)} \frac{p^{2}}{p \bar{p}+m^{2}}\left(t_{p}^{0}-t_{p}^{2}\right) \cdot I_{11}(p, m) .
$$

All terms are UV convergent, since they correspond to oversubtractions. The integral can be evaluated without further ado

$$
\left(t_{p}^{0}-t_{p}^{2}\right) \cdot I_{11}(p, m)=-m^{2} p^{2} \int \frac{d^{2} q}{(2 \pi)^{2}} \frac{q \bar{q}}{\left(q \bar{q}+m^{2}\right)^{4}}=\frac{i}{24 \pi} \frac{2}{m^{2}} p^{2},
$$

providing the following result for the difference (5.26)

$$
\Lambda_{2}(x-y)-\Lambda_{0}(x-y)=\frac{i \hbar^{2} D}{24 \pi m^{2}} \int \frac{\mathrm{d}^{2} p}{(2 \pi)^{2}} e^{i p \cdot(x-y)} \frac{p^{4}}{p \bar{p}+m^{2}} .
$$

After comparison with the rhs of (5.25) in the momentum representation,

$$
\rho\left\langle\partial^{3} c(x) b(y)\right\rangle_{c, 0}=-i \rho \hbar \int \frac{\mathrm{d}^{2} p}{(2 \pi)^{2}} e^{i p \cdot(x-y)} \frac{p^{4}}{p \bar{p}+m^{2}},
$$


the corresponding value for the contribution of the matter sector to the coefficient $\rho$ in eq. (5.25) is read off:

$$
\rho_{\mathrm{m}}=\frac{-\hbar D}{24 \pi m^{2}} .
$$

In the same way, the ghost sector contribution may now be evaluated by considering the double contractions between $T_{\mathrm{gh}}(x)$ and $\Theta_{\mathrm{gh}}(y)$ (5.23) in eq. (5.25), taking the factor $\left(\frac{1}{\partial} c\right)$ internal. The relevant BPHZ renormalized correlation functions $\tilde{\Lambda}_{a}(x-y)$ is

$$
\begin{aligned}
\tilde{\Lambda}_{a}(x-y) & =\frac{i}{\hbar} \int \mathrm{d}^{2} z\left\langle\mathrm{~N}_{2}[2 b \partial c+(\partial b) c](x) \mathrm{N}_{a}\left[\left(\frac{1}{\partial} b\right)\{(\partial c) c\}+\left(\frac{1}{\partial} c\right)\{2 b \partial c+(\partial b) c\}\right](z) b(y)\right\rangle_{c, 0} \\
& =-2 \hbar^{2} \int \frac{\mathrm{d}^{2} p}{(2 \pi)^{2}} e^{i p \cdot(x-y)} \frac{p}{p \bar{p}+m^{2}}\left(1-t_{p}^{a+1}\right) \cdot \tilde{I}(p, m),
\end{aligned}
$$

with the unrenormalized expression of the 1PI integral given by

$$
\tilde{I}(p, m)=\int \frac{\mathrm{d}^{2} q}{(2 \pi)^{2}} \frac{2 p^{2} q+q^{2} p-q^{3}}{\left(q \bar{q}+m^{2}\right)\left[(p-q)(\bar{p}-\bar{q})+m^{2}\right]} .
$$

The ghost sector contribution to the lhs of eq. (5.25) is the difference

$$
\tilde{\Lambda}_{2}(x-y)-\tilde{\Lambda}_{0}(x-y)=-2 \hbar^{2} \int \frac{\mathrm{d}^{2} p}{(2 \pi)^{2}} e^{i p \cdot(x-y)} \frac{p^{2}}{p \bar{p}+m^{2}}\left(t_{p}^{1}-t_{p}^{3}\right) \cdot \tilde{I}(p, m) .
$$

Keeping in the Taylor series for the integrand in (5.28) only those numerators that survive the $q$-integration, namely the ones of the form $(q \bar{q})^{n}$, yields

$$
\left(t_{p}^{1}-t_{p}^{3}\right) \cdot \tilde{I}(p, m)=\int \frac{\mathrm{d}^{2} q}{(2 \pi)^{2}}\left[\frac{2 q \bar{q}}{\left(q \bar{q}+m^{2}\right)^{3}}+\frac{(q \bar{q})^{2}}{\left(q \bar{q}+m^{2}\right)^{4}}-\frac{(q \bar{q})^{3}}{\left(q \bar{q}+m^{2}\right)^{5}}\right]=\frac{13 i}{24 \pi} \frac{p^{3}}{m^{2}} .
$$

The final result for $(5.29)$ is then

$$
\tilde{\Lambda}_{2}(x-y)-\tilde{\Lambda}_{0}(x-y)=\frac{-26 i \hbar^{2}}{24 \pi m^{2}} \int \frac{\mathrm{d}^{2} p}{(2 p i)^{2}} e^{i p \cdot(x-y)} \frac{p^{4}}{p \bar{p}+m^{2}}
$$

from which, by comparison with the momentum representation (5.27) of the rhs of eq. (5.25), the following value for the ghost sector contribution to the coefficient $\rho$ in eq. (5.25) is found

$$
\rho_{\mathrm{gh}}=\frac{26 \hbar}{24 \pi m^{2}} .
$$

The results can be summarized by giving the Zimmerman identity (5.22) explicitly as

$$
\mathrm{N}_{2}[\Theta(x)]-\mathrm{N}_{0}[\Theta(x)]=\frac{(26-D) \hbar}{24 \pi} \frac{1}{m^{2}} \mathrm{~N}_{2}\left[b^{*} \partial^{3} c(x)\right] .
$$

Inserted in eq. (5.21), it provides, after canceling the $m^{2}$ factors, the following form for the anomaly (5.20)

$$
\mathcal{A}=\frac{i(D-26)}{24 \pi} \int \mathrm{d}^{2} x c \partial^{3} b^{*}
$$

in full agreement with the result computed in the previous subsection (5.19).

In conclusion, the local form of the anomaly equation enabled us to trace back the source of the anomaly to the oversubtraction of the variation of a mass term. The remainder of this evanescent term in the massless limit was extracted through a Zimmerman identity. 


\section{The anomaly of chiral $W_{3}$ gravity}

In this section we first compute the anomaly of the chiral $W_{3}$ gravity model [37] using the anomalous Ward identities (4.7). Afterwards we also show how the two-loop contribution arises from (part of) the BRST breaking produced by the infrared regulating mass term, again through oversubtractions and Zimmerman's identity.

$W$-gravity models have recently attracted some attention for several reasons: as models with a non-linear symmetry algebra, as possible higher spin extensions of gravity, etc. For our purposes, the basic interest in the $W_{3}$ model lies in the fact that it is an example of a field theory with a genuine new contribution to the anomaly at the two-loop level. In other words, the rhs of (2.8) contains at the two-loop level not only nonlocal one-loop dressings of the one loop anomaly, but extra local two-loop contributions to the anomaly. The expression of the two-loop anomaly on the local level, eq.(4.9), rather than through its insertion in the effective action, adds extra clarity in that it is not necessary to disentangle it from the dressing of the one-loop anomaly. The expression we obtain for the anomaly in this model completely agrees with the existing literature [38, 39, 40, 41, 42, 21, 43, 5, 6]. Existing derivations of the two-loop contributions always involved -implicitly or explicitly- the use of OPE's of conformal field theory (for the one-loop anomaly, derivations using traditional field theory methods are found in [21, 5]). Our computation makes no difference between one and higher loop anomalies.

\subsection{The $W_{3}$ model}

Chiral $W_{3}$ gravity is the minimal higher spin extension of chiral $W_{2}$ gravity, in which matter fields couple to gauge fields through their spin 2 energy-momentum tensor $T$ and a spin 3 current $W$

$$
T=\frac{1}{2}\left(\partial \phi^{i}\right)\left(\partial \phi^{i}\right), \quad W=\frac{1}{3} d_{i j k}\left(\partial \phi^{i}\right)\left(\partial \phi^{j}\right)\left(\partial \phi^{k}\right) .
$$

The constants $d_{i j k}$ that determine the $W$ current are totally symmetric and satisfy

$$
d_{i(j k} d_{l) m i}=k \delta_{(j l} \delta_{k) m}
$$

for some arbitrary, but fixed parameter $k$.

The extra gauge symmetry generated by the spin 3 current $W$ is handled by supplementing the set of fields and antifields of chiral $W_{2}$ gravity with an extra ghost-antighost pair $(u, v)$, and their associated antifields. A tractable form of the extended actionO for chiral $W_{3}$ gravity in a gauge fixed basis can be taken from $21{ }^{2}$

$$
\begin{aligned}
S=\int \mathrm{d}^{2} x & \left\{\left[-\frac{1}{2}\left(\partial \phi^{i}\right)\left(\bar{\partial} \phi^{i}\right)+b(\bar{\partial} c)+v(\bar{\partial} u)\right]\right. \\
& +\phi_{i}^{*}\left[c\left(\partial \phi^{i}\right)+u d_{i j k}\left(\partial \phi^{j}\right)\left(\partial \phi^{k}\right)-2 k b(\partial u) u\left(\partial \phi^{i}\right)\right] \\
& +b^{*}[-T+2 b(\partial c)+(\partial b) c+3 v(\partial u)+2(\partial v) u] \\
& +v^{*}[-W+2 k T b(\partial u)+2 k \partial(T b u)+3 v(\partial c)+(\partial v) c] \\
& \left.+c^{*}[(\partial c) c+2 k T(\partial u) u]+u^{*}[2(\partial c) u-c(\partial u)]\right\} \\
= & S_{0}+\int \mathrm{d}^{2} x \Phi_{A}^{*}(x) R^{A}(x) .
\end{aligned}
$$

The antifields $b^{*}$ and $v^{*}$ take over the role of (minus) the gravitational gauge field and its spin 3 counterpart, respectively. The expressions multiplying these antifields in (6.2) are then (minus)

\footnotetext{
${ }^{20}$ See footnote 17

${ }^{21}$ The free parameter $\alpha$ that appears in 21 is taken to be zero here.
} 
the total energy momentum tensor $T_{t}$ and the total spin 3 current $W_{t}$. The extended action (6.2) shares an important property with (5.1), namely they are both of the form $S=S_{0}+\int \mathrm{N}_{2}\left[\Phi_{A}^{*} R^{A}\right]$, with $S_{0}$ purely quadratic in the fields: correlation functions for this model can therefore again be considered as perturbative series in the antifields.

The assignment of the relevant additive charges as in the $W_{2}$ model (Table 豆, are now completed with Table 2 for the extra fields.

\begin{tabular}{||c||c|c|c|c||}
\hline & $\operatorname{dim} d$ & $\operatorname{spin} j$ & gh. $\sharp$ & $d-j$ \\
\hline \hline$u$ & -2 & -2 & 1 & 0 \\
$v$ & 3 & 3 & -1 & 0 \\
\hline$u^{*}$ & 4 & 2 & -2 & 2 \\
$v^{*}$ & -1 & -3 & 0 & 2 \\
\hline
\end{tabular}

Table 2: Additive charges for fields and antifields of the spin 3 sector.

We finish the specification of the model by choosing the IR regulating mass term to be used in perturbative computations. For the matter fields the mass term (5.5) is used, such that the propagator is given by (5.6), whereas both for the spin $2(b, c)$ ghosts and for the new spin 3 $(u, v)$ ghosts a mass term of the form (5.7) is taken, leading to a propagator of the form (5.8) for both ghost pairs.

\section{2 $W_{3}$ anomalous Ward identities}

\subsubsection{Preliminary considerations}

We first analyze the anomalous Ward identities (4.7), as in the $W_{2}$ case, to find the general structure of the $W_{3}$ anomaly and describe the strategy we follow for its computation.

As for the $W_{2}$ case, it follows from Table 1 and Table 2 and the specific antifield dependence of the action that the BRST anomaly is linear in antifields, of the form $\mathcal{A}=\Phi_{A}^{*} F^{A}$. The equation from which one can derive information on the function $F^{A}$ is again of the form (5.9), in which the quantities $R^{A}$ should now be read off from the extended gauge-fixed action (6.2). Since loop diagrams come from at least double contractions between $R^{A}(x)$ and $R^{B}(y)$ on the lhs of (5.9), the fact that $R^{A}$ contains terms with up to four fields indicates that relevant diagrams for the computation of the anomaly may contain up to three loops. However, a closer look at the $\mathcal{O}\left(\Phi^{4}\right)$ terms in the $R^{A}$ 's, given by

$$
-2 k b(\partial u) u\left(\partial \phi^{i}\right), \quad 2 k[T b \partial u+\partial(T b u)], \quad 2 k[T(\partial u) u],
$$

and at the form of the propagators, shows that no proper three-loop diagram can arise formed by quartic contractions between the expressions in (6.3). The only two loop diagram arises from a triple contraction between two matter spin 3 currents $W$ (6.1), so that the potential two-loop anomaly depends only on the antifield $v^{*}$. Finally, double contractions between the different terms in the $R^{A}$ 's give rise to various one-loop contributions to the anomaly. Those are described in detail below. In summary, only one and two loop anomalies are possible in this model.

The antifield coefficients $F^{A}(\Phi)$ determining these one and two loop contributions to the anomaly are determined as in the $W_{2}$ case. First one selects those pairs $R^{A}(x), R^{B}(y)$ that admit double and triple contractions, and in a second step determines the "test product" of fields to be contracted with the remaining external legs. The precise form of the test product and a subsequent dimensional analysis completely determines the fields and the number of $\partial$-derivatives present in the coefficient $F^{A}$. The precise derivative structure and the numerical 
coefficients are obtained from an explicit (renormalized) computation of the lhs of (5.9). In doing this last step, the minimal normal ordering of the interactions prescribes minimal subtractions for all the divergent one loop integrals, which should therefore be substituted by their minimally renomalized counterpart (A.9). BPHZ renormalization of the relevant two-loop integral, requiring the use of Zimmerman's forest formula, will instead be treated in situ.

\subsubsection{One loop anomaly: a sample computation}

In this subsection, the procedure is illustrated by computing the "gravitational" part to the complete one loop BRST anomaly, viz. the contribution proportional to the antifield $b^{*}$. This suffices to get a flavour of the method. Further details on the computation of the full one loop anomaly can be found in appendix B.

Three different contributions to this gravitational part can be discerned: i) double contractions of $R^{b}=-T_{t}$ with itself; ii) contributions containing one factor $d_{i j k}$; and iii) contributions proportional to $k$, containing two such factors. We discuss them in detail in this order.

i) This is analogous to the computation in section 5. One uses $R^{b}=-\left(T+T_{2}+T_{3}\right)$, with $T_{j}$ defined by (5.3) with suitable substitution of $(b, c)$ by $(u, v)$ in $T_{3}$. Going through exactly the same steps as in sect. 5.2, but taking two contributions of the type (5.16) into account, namely for $j=2$ and for $j=3$, this first contribution to the one-loop anomaly of chiral $W_{3}$ gravity is

$$
\mathcal{A}_{1}^{(i)}=\frac{i(D-100)}{24 \pi} \int \mathrm{d}^{2} x c \partial^{3} b^{*} .
$$

ii) Contributions to the $b^{*}$-anomaly proportional to the tensor $d_{i j k}$ are generated by loops of matter fields coming from double contractions of $-T$ in $R^{b}$ with the terms

$$
\left\{\begin{array}{ccc}
u d_{i j k}\left(\partial \phi^{j}\right)\left(\partial \phi^{k}\right) & \text { in } & R^{\phi} \\
-\frac{1}{3} d_{i j k}\left(\partial \phi^{i}\right)\left(\partial \phi^{j}\right)\left(\partial \phi^{k}\right) & \text { in } & R^{v}
\end{array}\right.
$$

Obviously, a suitable test product for both contributions is $\phi^{i}(y) v(z)$, so that this part of the anomaly has to contain one factor $\phi^{i}$ and one factor $u$, while dimensional arguments require four derivatives $\partial$. For this contribution, the Ward identity (5.9) takes the form

$$
\begin{gathered}
\frac{i}{\hbar}\left\langle\mathrm{N}_{2}[-T(x)] \mathrm{N}_{0}\left[u d_{i j k}\left(\partial \phi^{j}\right)\left(\partial \phi^{k}\right)(y)\right] v(z)\right\rangle_{c, 0} \\
+\frac{i}{\hbar}\left\langle\mathrm{N}_{2}[-T(x)] \mathrm{N}_{3}\left[\frac{1}{3} d_{j k l}\left(\partial \phi^{j}\right)\left(\partial \phi^{k}\right)\left(\partial \phi^{l}\right)(z)\right] \phi^{i}(y)\right\rangle_{c, 0} \\
=-\alpha_{2}\left\langle\mathrm{~N}_{2}\left[F\left(\phi, u, \partial^{4} ; x\right)\right] \phi^{i}(y) v(z)\right\rangle_{c, 0},
\end{gathered}
$$

which can be represented by figure 2. These one-loop contributions are essentially the same as for the $W_{2}$. They are minimally subtracted, i.e. up to quadratic terms in the external momentum (see eq.([.4)). The resulting finite integrals are computed in eq.(A.9), and the lhs of (6.5) becomes

$$
\begin{aligned}
& \frac{\hbar^{2}}{12 \pi} d_{i l l} \int \frac{\mathrm{d}^{2} l}{(2 \pi)^{2}} \int \frac{\mathrm{d}^{2} q}{(2 \pi)^{2}} e^{-i l \cdot z} e^{-i q \cdot y} e^{i(l+q) \cdot x}\left[\frac{(l+q)^{3}}{(\bar{l}+\bar{q})} \frac{1}{\bar{l}}+\frac{(l+q)^{3}}{(\bar{l}+\bar{q})} \frac{1}{\bar{q}}\right]= \\
& \frac{\hbar^{2}}{12 \pi} d_{i l l} \int \frac{\mathrm{d}^{2} l}{(2 \pi)^{2}} \int \frac{\mathrm{d}^{2} q}{(2 \pi)^{2}} e^{-i l \cdot z} e^{-i q \cdot y} e^{i(l+q) \cdot x} \frac{(l+q)^{3}}{\bar{l} \bar{q}} .
\end{aligned}
$$



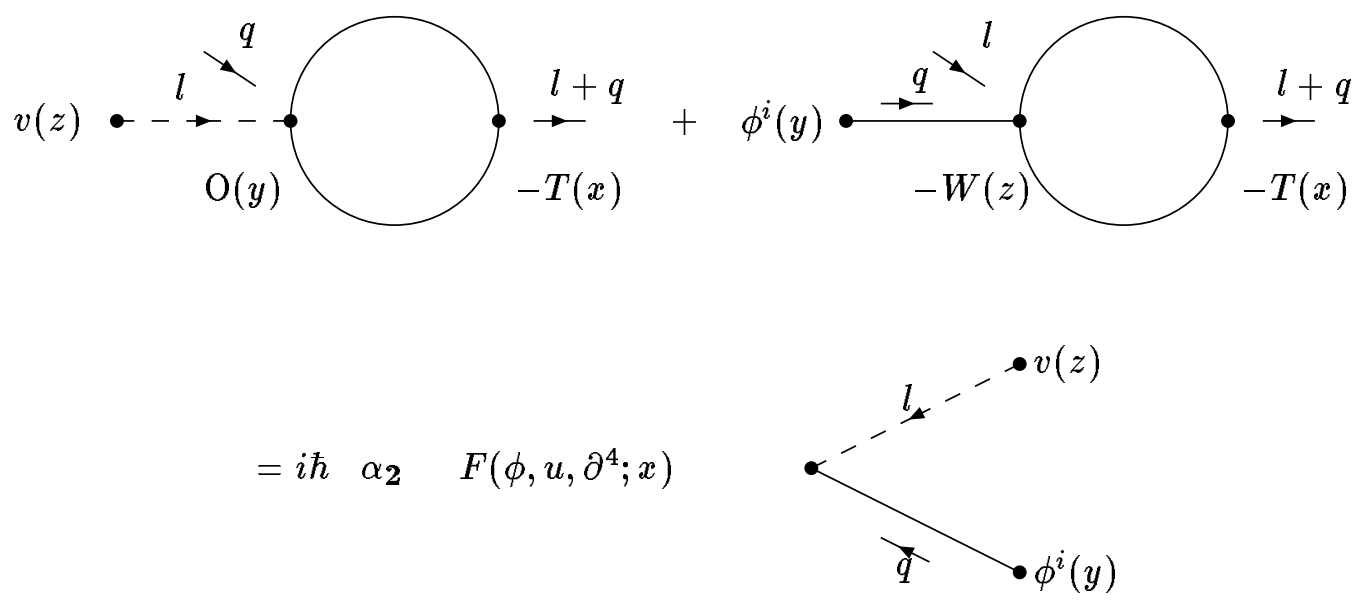

Figure 2: One-loop anomalous diagrams for $W_{3}$. The insertion in the first diagram is $O(y)=$ $\left[u d_{i j k}\left(\partial \phi^{j}\right)\left(\partial \phi^{i}\right)\right](y)$

This momentum dependence leads to the conclusion that in eq. 6.5) $F=\partial^{3}\left(u\left(\partial \phi^{i}\right) d_{i l l}\right)$, and determines the coefficient $\alpha_{2}$. This yields the second contribution to the anomaly:

$$
\mathcal{A}_{1}^{(i i)}=\frac{i}{24 \pi} \int \mathrm{d}^{2} x\left[2 u\left(\partial \phi^{k}\right) d_{i j k}\right] \partial^{3}\left[b^{*} \delta^{i j}\right] .
$$

iii) Contributions to the $b^{*}$-anomaly proportional to $k$ are generated by matter loops coming from two contractions of $-T$ in $R^{b}$ with the terms

$$
\left\{\begin{array}{clc}
2 k T b(\partial u)+2 k \partial(T b u) & \text { in } & R^{v} \\
2 k T(\partial u) u & \text { in } & R^{c}
\end{array}\right.
$$

A suitable test product for both contributions is now $v(y) v(z) c(t)$, yielding a possible anomaly containing two $u$ fields, one $b$ field and four derivatives $\partial$ in order to match the dimensions. (5.9) reads now

$$
\begin{aligned}
\left\{\frac { i } { \hbar } \left\langle\mathrm{N}_{2}[T(x)]\right.\right. & \left.\left.\mathrm{N}_{3}[2 k T b(\partial u)(y)+2 k \partial(T b u)(y)] v(z) c(t)\right\rangle_{c, 0}-(y \leftrightarrow z)\right\} \\
+ & \frac{i}{\hbar}\left\langle\mathrm{N}_{2}[T(x)] \mathrm{N}_{-1}[2 k T(\partial u) u(t)] v(y) v(z)\right\rangle_{c, 0} \\
= & -\alpha_{3}\left\langle\mathrm{~N}_{2}\left[F\left(b, u, u, \partial^{4} ; x\right)\right] v(y) v(z) c(t)\right\rangle_{c, 0} .
\end{aligned}
$$

The subtraction degrees computed from the $N_{a}$, once more the minimal ones, namely 2, yield a renormalized expression for the lhs of (6.7):

$$
\frac{\hbar^{3} i k D}{12 \pi} \int \frac{\mathrm{d}^{2} l}{(2 \pi)^{2}} \int \frac{\mathrm{d}^{2} q}{(2 \pi)^{2}} \int \frac{\mathrm{d}^{2} r}{(2 \pi)^{2}} e^{i(q+l+r) \cdot x} e^{-i q \cdot y} e^{-i r \cdot t} e^{-i l \cdot z} \frac{(q+l+r)^{3}(q-l)}{\bar{q} \bar{l} \bar{r}} .
$$


The momentum dependence determines $F=\partial^{3}(b u \partial u)$. After computing the numerical coefficient $\alpha_{3}$, the third contribution to the anomaly is

$$
\mathcal{A}_{1}^{(i i i)}=\frac{i}{24 \pi} \int \mathrm{d}^{2} x\left[-2 k b(\partial u) u \delta^{i j}\right] \partial^{3}\left[b^{*} \delta_{i j}\right] .
$$

In summary, collecting (6.4), (6.6) and (6.8), the gravitational part of the one-loop $W_{3}$ anomaly is

$$
\mathcal{A}_{1}^{b^{*}}=\mathcal{A}_{1, \mathrm{~m}}^{b^{*}}+\mathcal{A}_{1, \mathrm{gh}}^{b^{*}}=\frac{i}{24 \pi} \int \mathrm{d}^{2} x c^{i j} \partial^{3}\left(b^{*} \delta_{i j}\right)-\frac{100 i}{24 \pi} \int \mathrm{d}^{2} x c \partial^{3} b^{*},
$$

where we have defined

$$
c^{i j}=[c-2 k b(\partial u) u] \delta^{i j}+2 u d_{k}^{i j}\left(\partial \phi^{k}\right)
$$

for further use. The subscripts $m$ and gh will stand from now on for purely matter induced contributions and contributions induced by loops involving (one or two) ghost lines, respectively.

This procedure is applied time and again in appendix B in order to find the complete one-loop BRST anomaly. The final result is

$$
\mathcal{A}_{1}=\mathcal{A}_{1, \mathrm{~m}}+\mathcal{A}_{1, \mathrm{gh}}^{b^{*}}+\mathcal{A}_{1, \mathrm{gh}}^{\phi^{*}}+\mathcal{A}_{1, \mathrm{gh}}^{v^{*}},
$$

with $\mathcal{A}_{1, \mathrm{gh}}^{b^{*}}, \mathcal{A}_{1, \mathrm{gh}}^{\phi^{*}}$ and $\mathcal{A}_{1, \mathrm{gh}}^{v^{*}}$ given by (6.9), (B.3) and (B.7) respectively, and where $\mathcal{A}_{1, \mathrm{~m}}$ lumps together all the matter induced contributions in the compact expression

$$
\mathcal{A}_{1, \mathrm{~m}}=\frac{i}{24 \pi} \int \mathrm{d}^{2} x c^{i j} \partial^{3} h_{i j}^{*}
$$

with the "effective" antifield $h_{i j}^{*}$ given by

$$
h_{i j}^{*}=\left\{\delta_{i j}\left[b^{*}+2 k b\left(u\left(\partial v^{*}\right)-v^{*}(\partial u)\right)+2 k c^{*} u(\partial u)\right]-2 d_{i j}{ }^{k} \phi_{k}^{*} u+2 v^{*} d_{i j k}\left(\partial \phi^{k}\right)\right\} .
$$

Our result for the one-loop anomaly in the $W_{3}$ model is in perfect agreement with previous computations in the literature [39, 40, 41, 42, 21, 43, 5].

\subsubsection{Two-loop anomalous Ward identity}

After this brief discussion of the one loop anomaly, we now turn to the two loop anomaly. We consider it to be a major challenge for the present setup, since, as far as we know, no computation has been given that uses only the methods of renormalised perturbation theory.

As already pointed out, the unique two-loop diagram constructed from three contractions between two terms from the available $R^{A}$ is the diagram that corresponds to a triple contraction between two copies of the spin-3 current $W$. This immediately leads to a two loop anomaly proportional to $v^{*}$, since in the general Ward identity $(5.9)$ the $R$ 's are simply reduced to $W$. A suitable test product is hence $v(y)$, which on the rhs yields a function $F$ of the form $\alpha \partial^{5} u$, the only expression with non-vanishing correlation function with the test product $v$ and with the correct dimension 2. The Ward identity (5.9) takes the form

$$
\frac{i}{\hbar}\left\langle\mathrm{N}_{3}[W(x)] \mathrm{N}_{3}[W(y)]\right\rangle=\alpha\left\langle\mathrm{N}_{3}\left[\partial^{5} u(x)\right] v(y)\right\rangle \text {. }
$$

This is the analogue of $(\overline{5.10})$ in chiral $W_{2}$ gravity and corresponds to the triple contraction in the standard conformal field theory OPE of $W(x) W(y)$. 


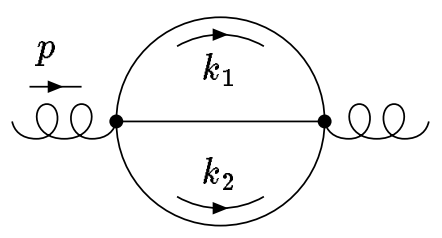

Figure 3: The anomalous two-loop diagram.

The computation of the lhs of (6.12), taking into account the appropriate number of subtractions, is a straightforward application of the forest formula. It is given explicitly in eq.(6.17). For readers unfamiliar with the rationale behind this forest, we build up the expression by considering the regions in momentum space which cause a divergence in the unsubtracted diagram.

The unregularized version of the two-loop integral $I^{R}(p, m)$ is given by

$$
I(p, m)=\int \frac{\mathrm{d}^{2} k_{1}}{(2 \pi)^{2}} \int \frac{\mathrm{d}^{2} k_{2}}{(2 \pi)^{2}} \mathbb{I}\left(k_{1}, k_{2} ; p, m\right),
$$

with the integrand

$$
\mathbb{I}\left(k_{1}, k_{2} ; p, m\right)=\frac{k_{1}^{2}}{k_{1} \bar{k}_{1}+m^{2}} \frac{k_{2}^{2}}{k_{2} \bar{k}_{2}+m^{2}} \frac{\left(p-k_{1}-k_{2}\right)^{2}}{\left[\left(p-k_{1}-k_{2}\right)\left(\bar{p}-\bar{k}_{1}-\bar{k}_{2}\right)+m^{2}\right]} .
$$

The momentum labeling is as in figure 3. In terms of the corresponding properly subtracted expression, the lhs of eq. (6.12) is

$$
-\frac{2}{3} d^{2} \hbar^{2} \int \frac{\mathrm{d}^{2} p}{(2 \pi)^{2}} e^{i p \cdot(x-y)} \mathbb{I}^{R}(p, m), \quad \text { with } \quad d^{2} \equiv d^{i j k} d_{i j k} .
$$

Thus we have to determine that expression. We start by identifying the possible superficial divergences. There are four regions in the integration domain where the integral potentially diverges:

$$
\begin{cases}k_{1} \rightarrow \infty, k_{2} \text { finite } & \text { quadratic divergence } \\ k_{2} \rightarrow \infty, k_{1} \text { finite } & \text { quadratic divergence } \\ k_{1} \rightarrow \infty, k_{2} \rightarrow \infty, k_{1}+k_{2} \text { finite } & \text { quadratic divergence } \\ k_{1} \rightarrow \infty, k_{2} \rightarrow \infty & \text { quartic divergence. }\end{cases}
$$

The quadratically divergent regions are associated with the one-loop subdiagrams $\gamma_{i}, i=1,2,3$ -pictorially represented in figure 4 - obtained by cutting one of the three internal lines between $W(x)$ and $W(y)$ in the original two-loop diagram (we take $\gamma_{1}$ to correspond to cutting the $k_{2}$ line, $\gamma_{2}$ the $k_{1}$ line, and $\gamma_{3}$ the $\left(p-k_{1}-k_{2}\right)$ line). The number of subtractions is, as always, determined from (5.4), and amounts to the minimal subtraction of the quadratic subdivergences identified above. The cut line corresponds to a field $\partial \phi$, and if we include the resulting momentum dependence of the numerator (but not the propagator) in the expresssion for the subdiagram, the subtraction degree is 4 . The quartic divergence of the complete diagram $G$ also has subtraction degree 4. Zimmerman's forest formula [34 then consists of two steps. In the first step, the superficial quadratic divergences in the original integrand associated with the subdiagrams $\gamma_{i}$ are subtracted:

$$
\mathbb{I}-\sum_{i=1}^{3} t_{p_{\gamma_{i}}}^{4} \mathcal{I}\left(\gamma_{i}\right),
$$



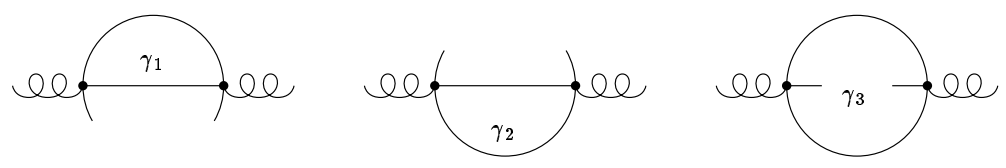

Figure 4: The 3 one-loop divergent subgraphs.

where $p_{\gamma_{i}}$ stands for the external momenta of the $\gamma_{i}$ subdiagram and $\mathbb{I}\left(\gamma_{i}\right)$ for the suitable restriction of the original integrand (6.14) to the subdiagram $\gamma_{i}$. This leads to an expression that is still quartically divergent. A further quartic subtraction cures this:

$$
\left(1-t_{p}^{4}\right)\left[\mathcal{I}-\sum_{i=1}^{3} t_{p_{\gamma_{i}}}^{4} \mathcal{I}\left(\gamma_{i}\right)\right] .
$$

The net result of this process is Zimmerman's forest formula adapted to our integrand (6.14)

$$
\mathbb{I}-t_{p}^{4} \mathbb{I}-\sum_{i=1}^{3} t_{p_{\gamma_{i}}}^{4} \mathbb{I}+\sum_{i=1}^{3} t_{p_{p}^{4}}^{4} t_{p_{\gamma_{i}}}^{4} \mathbb{I}=\sum_{\mathcal{U} \in \mathcal{F}} \prod_{\gamma \in \mathcal{U}}\left(-t_{p_{\gamma}}^{d(\gamma)}\right) \mathbb{I},
$$

where $\mathcal{F}$ stands for the set of all possible forests $\mathcal{U}$ of the two-loop diagram, namely

$$
\mathcal{F}=\left\{\emptyset,\{G\},\left\{\gamma_{1}\right\},\left\{\gamma_{2}\right\},\left\{\gamma_{3}\right\},\left\{\gamma_{1}, G\right\},\left\{\gamma_{2}, G\right\},\left\{\gamma_{3}, G\right\}\right\}
$$

Expression (6.17) is the subtracted integrand which upon substitution in (6.13) yields the proper expression of our two-loop integral.

A closer inspection of (6.17) reveals that in its last two sums the terms corresponding to $i=1,2$ cancel each other. We exemplify this for $\gamma_{1}$, i.e. $\left(1-t_{p}^{4}\right) t_{p_{\gamma_{1}}}^{4} \mathbb{I}$. The expression of the integrand (6.14) restricted to the subdiagram $\gamma_{1}$ reads

$$
\mathbb{I}\left(\gamma_{1}\right)=\frac{r_{2}^{2}}{k_{2} \bar{k}_{2}+m^{2}} \frac{k_{1}^{2}}{k_{1} \bar{k}_{1}+m^{2}} \frac{\left(p+r_{1}-k_{1}\right)^{2}}{\left[\left(p+r_{1}-k_{1}\right)\left(\bar{p}+\bar{r}_{1}-\bar{k}_{1}\right)+m^{2}\right]},
$$

where in the set of external momenta, $p_{\gamma_{1}}=\left\{p, r_{1}\right\}, r_{1}$ must be identified with $-k_{2}$ after having done the Taylor expansion. In this way, the contribution generated by the forest $\left\{\gamma_{1}\right\}$ is

$$
\left.t_{p_{\gamma_{1}}}^{4} \mathbb{I}\left(\gamma_{1}\right)\right|_{r_{1}=-k_{2}}=\frac{k_{2}^{2}}{k_{2} \bar{k}_{2}+m^{2}} \frac{k_{1}^{2}}{k_{1} \bar{k}_{1}+m^{2}} \mathcal{O}\left(p^{2}\right)
$$

where $\mathcal{O}\left(p^{2}\right)$ stands for a polynomial of degree two in the momentum $p$ whose coefficients are rational functions of $k_{1}$ and $k_{2}$. It is then evident that the action of $\left(1-t_{p}^{4}\right)$ on (6.18) produces a vanishing result, since on quadratic polynomials in $p, t_{p}^{4}$ acts like the identity. The same mechanism can be seen to hold as well for the subdiagram $\gamma_{2}$, obtained from $\gamma_{1}$ upon interchange of $k_{1}$ and $k_{2}$.

For $\gamma_{3}$ the situation is slightly different. Indeed, the Taylor series expansion in the external momenta $p_{\gamma_{3}}=\left\{p, r_{3}\right\}$ of the integrand (6.14) adapted to $\gamma_{3}$, i.e.

$$
\mathbb{I}\left(\gamma_{3}\right)=\frac{k_{2}^{2}}{k_{2} \bar{k}_{2}+m^{2}} \frac{r_{3}^{2}}{\left[\left(p+r_{3}-k_{2}\right)\left(\bar{p}+\bar{r}_{3}-\bar{k}_{2}\right)+m^{2}\right]} \frac{\left(p+r_{3}-k_{2}\right)^{2}}{\left[\left(p-k_{1}-k_{2}\right)\left(\bar{p}-\bar{k}_{1}-\bar{k}_{2}\right)+m^{2}\right]},
$$


yields a nonpolynomial dependence on the external momentum $p$, namely

$$
\begin{gathered}
\left.t_{p_{\gamma_{3}}}^{4} \mathcal{I}\left(\gamma_{3}\right)\right|_{r_{3}=\left(-p+k_{1}+k_{2}\right)}= \\
\left.\frac{k_{2}^{2}}{k_{2} \bar{k}_{2}+m^{2}} \frac{\left(p-k_{1}-k_{2}\right)^{2}}{\left[\left(p-k_{1}-k_{2}\right)\left(\bar{p}-\bar{k}_{1}-\bar{k}_{2}\right)+m^{2}\right]} t_{p_{\gamma_{3}}}^{2}\left[\frac{\left(p_{\gamma_{3}}-k_{2}\right)^{2}}{\left[\left(p_{\gamma_{3}}-k_{2}\right)\left(\bar{p}_{\gamma_{3}}-\bar{k}_{2}\right)+m^{2}\right]}\right]\right|_{p_{\gamma_{3}}=\left(k_{1}+k_{2}\right)} .
\end{gathered}
$$

This produces a nonvanishing result when the operator $\left(1-t_{p}^{4}\right)$ acts on it. Using the more convenient integration variables $l=k_{1}+k_{2}$ and $k=k_{2}$, the renormalized expression of the two-loop integral (6.13) is thus found to be

$$
\begin{aligned}
I^{R}(p, m)= & \int \frac{\mathrm{d}^{2} l}{(2 \pi)^{2}}\left(1-t_{p}^{4}\right) \frac{(p-l)^{2}}{\left[(p-l)(\bar{p}-\bar{l})+m^{2}\right]} \times \\
& \int \frac{\mathrm{d}^{2} k}{(2 \pi)^{2}}\left(1-t_{l}^{2}\right)\left[\frac{k^{2}}{k \bar{k}+m^{2}} \frac{(l-k)^{2}}{\left[(l-k)(\bar{l}-\bar{k})+m^{2}\right]}\right] .
\end{aligned}
$$

It is now a straightforward task to evaluate (6.19). Indeed, since the $k$-"one-loop" integral is precisely of the minimally subtracted form A.1, A.2 considered in appendix A, with $a=b=2$, use of A.8,A.9) brings it to the form

$$
I^{R}(p, m)=\frac{-i}{12 \pi} \int \frac{\mathrm{d}^{2} l}{(2 \pi)^{2}}\left(1-t_{p}^{4}\right)\left[F\left(\frac{m^{2}}{l \bar{l}}\right) \frac{l^{4}}{l \bar{l}+m^{2}} \frac{(p-l)^{2}}{[(p-l)(\bar{p}-\bar{l})]+m^{2}}\right] .
$$

Apart from the extra factor $F\left(\frac{m^{2}}{l l}\right)$, with $F(0)=1$, this is of the same generic minimally subtracted form (A.1, A.2), now with $a=4, b=2$. Therefore, in the limit of interest $m \rightarrow 0$, the one-loop result (A.9) can again be used, yielding for 6.20

$$
I^{R}(p, 0)=-\frac{1}{480 \pi^{2}} \frac{p^{5}}{\bar{p}}
$$

and for the lhs (6.15) of the Ward identity (6.12) the final result

$$
\frac{d^{2} \hbar^{2}}{720 \pi^{2}} \int \frac{\mathrm{d}^{2} p}{(2 \pi)^{2}} e^{i p \cdot(x-y)} \frac{p^{5}}{\bar{p}}
$$

Comparing the expression (6.21) with the rhs of (6.12)

$$
i \alpha \hbar \int \frac{\mathrm{d}^{2} p}{(2 \pi)^{2}} e^{i p \cdot(x-y)} \frac{p^{5}}{\bar{p}},
$$

the value of the coefficient $\alpha$ is read off, and the complete form of the $W_{3}$ two-loop anomaly is

$$
\mathcal{A}_{2}=\frac{i \hbar d^{2}}{720 \pi^{2}} \int \mathrm{d}^{2} x u \partial^{5} v^{*}
$$

in agreement with [40, 42, 6]. This concludes our analysis of the one- and two-loop anomalies in the $W_{3}$ gravity model by means of the anomalous Ward identities.

\subsection{The $W_{3}$ two-loop anomaly from the mass term}

We show in this section how the two-loop contribution (6.22) to the BRST anomaly for $W_{3}$ arises as a consequence of the explicit BRST symmetry breaking by the IR regulating mass 
term, i.e. from the eq. (5.20). The main tool is again the reduction of the oversubtracted BRST variation of the mass term through the use of Zimmerman identities.

The $W_{3}$ IR regulating mass term is formed by adding (5.5), (5.7) and a copy of the latter for the $u v$ ghost system. Its BRST variation is

$$
\begin{gathered}
\mathrm{N}_{2}\left[\frac{\overleftarrow{\delta} S_{m}}{\delta \Phi^{A}(x)}\left\{\frac{\vec{\delta} S}{\delta \Phi_{A}^{*}(x)}\right\}\right]= \\
-m^{2} \mathrm{~N}_{2}\left[\phi^{i}(x)\left\{c\left(\partial \phi^{i}\right)+u d_{i j k}\left(\partial \phi^{j}\right)\left(\partial \phi^{k}\right)-2 k b(\partial u) u\left(\partial \phi^{i}\right)\right\}\right] \\
+m^{2} \mathrm{~N}_{2}\left[\left(\frac{1}{\partial} b\right)\left\{R^{c}\right\}+\left(\frac{1}{\partial} c\right)\left\{R^{b}\right\}+\left(\frac{1}{\partial} v\right)\left\{R^{u}\right\}+\left(\frac{1}{\partial} u\right)\left\{-W-W_{\text {gh }}\right\}\right]
\end{gathered}
$$

When inserted in loop correlation functions, it gives rise to a multitude of one-loop diagrams upon double contractions with the interaction terms $\Phi_{A}^{*} R^{A}$ in (6.2). These diagrams are of course the analogues of the one-loop correlation functions studied in sect.6.2.2 and in appendix B. We will not dwell on a rederivation of the one loop contribution to the $W_{3}$ anomaly, but concentrate instead on the more interesting two-loop contribution in this approach. Just as before, two-loop contributions can only be generated by triple matter contractions. These occur now between the interaction term $-v^{*} \mathrm{~N}_{3}[W]$ and the oversubtracted $\phi^{3}$ type terms in (6.23), namely

$$
-m^{2} \mathrm{~N}_{2}\left[\phi^{i}\left\{u d_{i j k}\left(\partial \phi^{j}\right)\left(\partial \phi^{k}\right)\right\}\right], \quad \text { and } \quad-m^{2} \mathrm{~N}_{2}\left[\left(\frac{1}{\partial} u\right)\{W\}\right] .
$$

Both contractions leave a factor $u$ free and the two-loop anomaly is therefore necessarily of the form $\mathcal{A}_{2}=m^{-2} \tilde{\rho} v^{*} \partial^{5} u$, in accordance with the analysis presented in sect. 6.2.3. Anisotropy forces the second term in (6.24) to behave effectively as a minimally subtracted, isotropic normal product, since the factor $\partial^{-1} u$ is always in an external line. Upon substitution in eq. (5.20), no two-loop contribution is obtained from this term. The two-loop anomaly can then be extracted from the the Zimmerman identity

$$
\mathrm{N}_{2}\left[\phi^{i}\left\{u d_{i j k}\left(\partial \phi^{j}\right)\left(\partial \phi^{k}\right)\right\}(x)\right]-\mathrm{N}_{0}\left[\phi^{i} u d_{i j k}\left(\partial \phi^{j}\right)\left(\partial \phi^{k}\right)(x)\right]=\rho \mathrm{N}_{2}\left[v^{*} \partial^{5} u\right]+\ldots
$$

More specifically, the relevant coefficient will arise from a two-loop calculation. We define

$$
\Sigma_{a}(x-y)=-\frac{i}{\hbar} \int \mathrm{d}^{2} z\left\langle\mathrm{~N}_{3}[W(x)] \mathrm{N}_{a}\left[\phi^{i}\left\{u d_{i j k}\left(\partial \phi^{j}\right)\left(\partial \phi^{k}\right)\right\}(z)\right] v(y)\right\rangle_{c, 0} .
$$

The coefficient $\rho$ in $(6.25)$ is then determined by

$$
\Sigma_{2}(x-y)-\Sigma_{0}(x-y)=\rho\left\langle\mathrm{N}_{3}\left[\partial^{5} u(x)\right] v(y)\right\rangle_{c, 0} .
$$

The unsubtracted 1PI two-loop integral corresponding to (6.26), is

$$
2 i \hbar^{3} d^{2} \int \frac{\mathrm{d}^{2} p}{(2 \pi)^{2}} e^{i p \cdot(x-y)} \frac{p}{p \bar{p}+m^{2}} \widetilde{I}(p, m),
$$

where the integrand is

$$
\begin{gathered}
\widetilde{I}(p, m)=\int \frac{\mathrm{d}^{2} k_{1}}{(2 \pi)^{2}} \int \frac{\mathrm{d}^{2} k_{2}}{(2 \pi)^{2}} \widetilde{\mathbb{I}}\left(k_{1}, k_{2} ; p, m\right) \\
=\int \frac{\mathrm{d}^{2} k_{1}}{(2 \pi)^{2}} \int \frac{\mathrm{d}^{2} k_{2}}{(2 \pi)^{2}} \frac{k_{1}^{2}}{k_{1} \bar{k}_{1}+m^{2}} \frac{k_{2}^{2}}{k_{2} \bar{k}_{2}+m^{2}} \frac{\left(p-k_{1}-k_{2}\right)}{\left[\left(p-k_{1}-k_{2}\right)\left(\bar{p}-\bar{k}_{1}-\bar{k}_{2}\right)+m^{2}\right]},
\end{gathered}
$$

\footnotetext{
${ }^{22}$ Note that for minimally subtracted normal products, anisotropic and isotropic versions coincide.
} 
which differs from the previous two-loop integral (6.14) in one factor $\left(p-k_{1}-k_{2}\right)$. This changes the analysis of the divergent integration domains slightly in comparison with (6.16). Using the same momentum labeling, one has

$$
\begin{cases}k_{1} \rightarrow \infty, k_{2} \text { finite } & \text { linear divergence } \\ k_{2} \rightarrow \infty, k_{1} \text { finite } & \text { linear divergence } \\ k_{1} \rightarrow \infty, k_{2} \rightarrow \infty, k_{1}+k_{2} \text { finite } & \text { quadratic divergence } \\ k_{1} \rightarrow \infty, k_{2} \rightarrow \infty & \text { cubic divergence. }\end{cases}
$$

The same labeling for the three different subdiagrams as in the previous subsection is used. In particular, $\gamma_{3}$ corresponds to the quadratic divergence in (6.30). The subtraction for the full two-loop diagram is always isotropic, and the degree is $2+(3-2)+(a-2)+(3-1)=3+a$. For the subdiagrams $\gamma_{1}$ and $\gamma_{2}$, the $\phi^{i}$ is always in the loop itself, and the subtraction degrees are the same as for the two-loop diagram. For the third one-loop subdiagram $\phi^{i}$ is an external line, and the prescribed subtraction is always the minimal subtraction to order 3 , for both values of a. A straigtforward application of Zimmerman's forest formula on the integral $(6.28,6.29)$ yields for the integrand determining the difference $\Sigma_{2}-\Sigma_{0}(6.27)$ the symbolic expression

$$
\left(t_{p}^{3}-t_{p}^{5}\right)\left(1-t_{p_{\gamma_{3}}}^{3}\right) \cdot \tilde{\mathbb{I}}+\sum_{i=1,2} t_{p}^{5}\left(1-t_{p_{\gamma_{i}}}^{5}\right) \cdot \tilde{\mathbb{I}}-\sum_{i=1,2} t_{p}^{3}\left(1-t_{p_{\gamma_{i}}}^{3}\right) \cdot \tilde{\mathbb{I}}
$$

The contributions related to $\gamma_{1}$ and $\gamma_{2}$, namely the last two terms in (6.31), are identically zero by exactly the same argument that was given in the previous subsection (cf. eq. $(6.18)$ ). The difference in eq. (6.27) is

$$
\Sigma_{2}(x-y)-\Sigma_{0}(x-y)=2 i \hbar^{3} d^{2} \int \frac{\mathrm{d}^{2} p}{(2 \pi)^{2}} e^{i p \cdot(x-y)} \frac{p}{p \bar{p}+m^{2}} \widetilde{I}_{R}(p, m),
$$

with the 1PI part given by

$$
\begin{aligned}
\widetilde{I}_{R}(p, m)= & \int \frac{\mathrm{d}^{2} l}{(2 \pi)^{2}}\left(t_{p}^{3}-t_{p}^{5}\right) \frac{(p-l)}{\left[(p-l)(\bar{p}-\bar{l})+m^{2}\right]} \times \\
& \int \frac{\mathrm{d}^{2} k}{(2 \pi)^{2}}\left(1-t_{l}^{2}\right)\left[\frac{k^{2}}{k \bar{k}+m^{2}} \frac{(l-k)^{2}}{\left[(l-k)(\bar{l}-\bar{k})+m^{2}\right]}\right] .
\end{aligned}
$$

The same integration variables as in (6.19) were used. The consequence of the anisotropic normal product is that the subtraction of the one loop subdivergences is the minimal one. For the overall subtraction, there is a difference and the entire two-loop anomaly is due to this.

We now compute $\widetilde{I}_{R}$. Using eq.(A.8) for the one-loop, minimally subtracted $k$ subintegral, we obtain

$$
\widetilde{I}_{R}(p, m)=-\frac{i}{2 \pi} \int_{0}^{1} d x x^{2}(1-x)^{2} \int \frac{\mathrm{d}^{2} l}{(2 \pi)^{2}}\left(t_{p}^{3}-t_{p}^{5}\right) \frac{(p-l)}{\left[(p-l)(\bar{p}-\bar{l})+m^{2}\right]}\left[\frac{l^{4}}{x(1-x) l \bar{l}+m^{2}}\right] .
$$

Only the terms of the form $(l \bar{l})^{n}$ in the difference between the Taylor series survive the $l$ angular integral, such that $(6.33)$ becomes

$$
\widetilde{I}_{R}(p, m)=\frac{i}{2 \pi} p^{5} m^{2} \int_{0}^{1} d x x^{2}(1-x)^{2} \int \frac{\mathrm{d}^{2} l}{(2 \pi)^{2}}\left[\frac{1}{x(1-x) l \bar{l}+m^{2}}\right] \frac{(l \bar{l})^{4}}{\left(l \bar{l}+m^{2}\right)^{6}} .
$$

The Wick rotated $l$ momentum integral is conveniently performed using polar coordinates, and yields after changing radial integration variable to $z=|l|^{2} / 2 m^{2}$,

$$
\widetilde{I}_{R}(p, m)=\frac{1}{4 \pi^{2}} \frac{p^{5}}{m^{2}} \int_{0}^{1} d x x^{2}(1-x)^{2} \int_{0}^{\infty} d z \frac{z^{4}}{(z+1)^{6}[x(1-x) z+1]} .
$$


A further change of variables $z=t /(1-t)$, followed by the change to $\left\{\alpha_{1}=t x, \alpha_{2}=t(1-x), \alpha_{3}=1-t\right\}$ brings it in the more familiar form

$$
\begin{aligned}
\widetilde{I}_{R}(p, m) & =\frac{1}{4 \pi^{2}} \frac{p^{5}}{m^{2}}\left(\prod_{i} \int_{0}^{1} d \alpha_{i}\right) \delta\left(\sum_{i=1}^{3} \alpha_{i}-1\right) \frac{\alpha_{1}^{2} \alpha_{2}^{2} \alpha_{3}}{\alpha_{1} \alpha_{2}+\alpha_{1} \alpha_{3}+\alpha_{2} \alpha_{3}} \\
& =\frac{1}{4 \pi^{2}} \frac{p^{5}}{m^{2}} \frac{1}{3}\left(\prod_{i} \int_{0}^{1} d \alpha_{i}\right) \delta\left(\sum_{i=1}^{3} \alpha_{i}-1\right) \alpha_{1} \alpha_{2} \alpha_{3},
\end{aligned}
$$

where the last line is obtained by symmetrising the previous numerator in the $\alpha_{i}$. Further use of the well-known result

$$
\left(\prod_{i=1}^{n} \int_{0}^{1} d \alpha_{i}\right) \delta\left(\sum_{i=1}^{n} \alpha_{i}-1\right) \prod_{i=1}^{n} \alpha_{i}^{a_{i}-1}=\frac{\prod_{i=1}^{n} \Gamma\left(a_{i}\right)}{\Gamma\left(\sum_{i} a_{i}\right)}
$$

gives

$$
\widetilde{I}_{R}=\frac{1}{2 \pi^{2}} \frac{p^{5}}{m^{2}} \frac{1}{6 !}
$$

When plugged back in (6.27, 6.32) this determines the coefficient $\rho$ in the Zimmerman identity (6.25):

$$
\rho=-\frac{d^{2} \hbar^{2}}{6 ! \pi m^{2}}
$$

Insertion of this Zimmerman identity in eq. (6.23) leads then to the cancellation of its overall $m^{2}$ factor with the $m^{-2}$ factor in (6.34) and finally gives a nonvanishing two-loop contribution to the anomaly $(5.20)$ in the limit $m \rightarrow 0$. We find

$$
\mathcal{A}_{2}=\frac{i \hbar d^{2}}{720 \pi^{2}} \int \mathrm{d}^{2} x u \partial^{5} v^{*}
$$

which is in complete agreement with the previous result (6.22).

\section{Conclusions and Outlook}

In this paper, we have started a program to put the quantum theory of the BV quantisation formalism on a sounder basis. The old machinery of BPHZ renormalisation and normal ordered products turns out to be well suited for this purpose. We have given a formulation of the quantum BV master equation using these normal ordered products, which is valid to all orders in perturbation theory. An advantage over the Zinn-Justin formulation in terms of the effective action is that we work with local operator equations. As a concrete testing ground, we have computed the BRST anomalies for some two dimensional field theories, where it is known that, in contrast with Yang-Mills theories, new anomalies appear in higher loops.

Apart from the possibility to compute the anomaly - to all orders in perturbation theory directly from the Veltman-Ward identity, a closed local expression for the anomaly was obtained (eq.(4.9)). Two ingredients are of crucial importance here. First, to apply the BPHZ method to massless theories one should temporarily introduce a mass term: the anomaly is related to the explicit breaking of the BRST symmetry by this mass term. This is reminiscent of, but different from, the one-loop Pauli-Villars method. The second ingredient is the use of anisotropic oversubtracted normal ordered operators. They originate in the mass term, and occur with a coefficient proportional to the mass (squared). The massless limit does not vanish however: it is extracted by rewriting the oversubtracted operator in terms of a set of minimally subtracted ones, using Zimmerman's normal product identity. The anomaly is what remains in this limit. 
We have successfully applied our method to the computation of the anomalies in $W_{2}$ and $W_{3}$ gravity. In particular, detecting the presence of the two-loop anomaly of the latter model is as easy as detecting the one-loop anomaly of either model. Only the computation of the coefficient, involving the renormalisation of a two-loop diagram using forest formula techniques, is more involved.

Of course, these methods can also be applied to compute anomalies in global symmetries. As an example, we verified that with our choice of mass term, the correct ghost number anomaly is reproduced. Despite the fact that both the original action and the mass term have ghost number zero, the associated Noether current is not conserved, owing to a non-locality in the mass term, and again the occurrence of oversubtracted operators.

Having demonstrated the possibilities offered by the BPHZ method in setting up proper renormalised versions of the quantum master equation, we now give some possible directions for further development.

A straightforward extension would be to include extra finite "counterterms" in the action. In specific examples, these harbour the possibility to cancel or shift the anomalies of the quantum theory. These are related to the possibility that the interaction terms and/or the BRST transformation rules are not minimally subtracted, and presumably also to the choice of mass term, as in Pauli-Villars regularisation. Also, whereas our local anomaly equation is in principle valid irrespective of the closing of the algebra off-shell, it could be of interest to work out explicitly an example where the extended action is non-linear in the antifields.

On a more theoretical level the question of consistency conditions for higher loop anomalies imposes itself. For the effective action we have that $(\Gamma, \mathcal{A} \cdot \Gamma)=0$. Formally, it is easy to derive the corresponding local conditions perturbatively in $\hbar$, but unfortunately this is again ill-defined due to the use of the formal operator $\Delta$ (see section 2). To lowest order, this corresponds to the condition of Wess and Zumino that the one-loop anomalies are consistent, i.e. BRST closed. In the BPHZ formulation that we gave, the offending $\Delta$ is absent ${ }^{23}$. The question of higher-loop Wess -Zumino condition is therefore open. Our local anomaly formula, eq.(4.9), suggests that the normal product equations could be rather simple transcriptions of the classical equations, provided one uses the appropriate anisotropic versions.

The definition of the normal products takes as its starting point a set of well-defined Feynman rules. This means, in BV, that one starts from a certain gauge fixed basis. It would be interesting to find a formulation that does not depend on this choice, or at least to show how the transition from one choice to another one can be made. This poses of course the problem of treating canonical transformations (in the antibracket sense) on the quantum level with the BPHZ method. We recall that within the BV scheme, a change of gauge is implemented via a canonical transformation. Possible counterterms that are generated by this are also related to the Jacobian of the BRST transformation. Although for a closed algebra it is formally independent of the gauge, in a regularised computation ${ }^{24}$ one may obtain a gauge dependent expression, owing to the gauge dependence of the regulator.

The final task would be the unraveling of the quantum cohomology. On the non-local level, the quantum BRST transformation can be defined as $(\Gamma, X)=\mathcal{S} X$. For non-anomalous theories, this is a nilpotent operator. It can be translated back formally to the level of local field theory, where the quantum BRST operator is $\sigma X=(X, W)-i \hbar \Delta X$, again involving the ill-defined $\Delta$. One may hope that the normal products of Zimmerman will enable one to define a replacement for this operation, not just for the action (this is what the anomaly gives), but also for arbitrary functionals of fields and antifields.

\footnotetext{
${ }^{23}$ Another possibility is to stay with $\Delta$, but de-localise all the functionals, as in the nonlocal regularisation scheme treated in [5]. See $[6]$ for further developments in this direction.

${ }^{24}$ We refer to the case of Yang-Mills regularised with Pauli-Villars, see 44].
} 


\section{Acknowledgements}

It is a pleasure to thank Fernando Ruiz Ruiz for discussions on various aspects of regularization and renormalization. F. D. J. is supported by the Human Capital and Mobility Programme through the network on Constrained Dynamical Systems. J.P. acknowledges financial support from the Spanish ministry of education (MEC).

\section{A BPHZ renormalized one-loop integrals}

Our main goal in this appendix is to compute the general form of the BPHZ renormalized, minimally subtracted, one-loop integrals used throughout the paper.

At the one-loop level, the specific form of the interactions for chiral $W_{2}$ and $W_{3}$ gravity leads to connected Green's functions which always contain as a 1PI part a special case of the general one-loop integral $I_{a b}(p, m)(5.13)$

$$
I_{a b}(p, m)=\int \frac{\mathrm{d}^{2} q}{(2 \pi)^{2}} \frac{q^{a}(p-q)^{b}}{\left(q \bar{q}+m^{2}\right)\left[(p-q)(\bar{p}-\bar{q})+m^{2}\right]} \equiv \int \frac{\mathrm{d}^{2} q}{(2 \pi)^{2}} \mathcal{I}_{a b}(q, p, m),
$$

with $a, b \geq 1$. With a superficial degree of divergence given by $a+b-2$, minimal subtraction prescribes that these integrals are replaced by

$$
I_{a b}^{R}(p, m)=\left[\left(1-t_{p}^{a+b-2}\right) \cdot I_{a b}(p, m)\right]
$$

using the shorthand (5.12).

First one expresses the propagators in (A.2) by means of their well-known integral representation $a^{-1}=\int_{0}^{\infty} d t e^{-a t}$, i.e.

$$
I_{a b}^{R}(p, m)=\int \frac{\mathrm{d}^{2} q}{(2 \pi)^{2}}\left(1-t_{p}^{a+b-2}\right) \int_{0}^{\infty} d t_{1} \int_{0}^{\infty} d t_{2} e^{-t_{1}\left(q \bar{q}+m^{2}\right)} e^{-t_{2}\left[(p-q)(\bar{p}-\bar{q})+m^{2}\right]} q^{a}(p-q)^{b} .
$$

After changing integration variables to $t=t_{1}+t_{2}$ and $x=t_{2} / t$, this becomes

$$
I_{a b}^{R}(p, m)=\int \frac{\mathrm{d}^{2} q}{(2 \pi)^{2}} \int_{0}^{\infty} \mathrm{d} t t \int_{0}^{1} \mathrm{~d} x e^{-t\left(q \bar{q}+m^{2}\right)}\left(1-t_{p}^{a+b-2}\right)\left[q^{a}(p-q)^{b} e^{-t x[p \bar{p}-q \bar{p}-\bar{q} p]}\right] .
$$

For the actual computation of this integral it is not necessary to know the subtraction terms in detail, the knowledge of their general form is sufficient. The Taylor series expansion around vanishing external momenta of the relevant factor of the integrand in (A.3) is

$$
\begin{gathered}
q^{a}(p-q)^{b} e^{-t x[p \bar{p}-q \bar{p}-\bar{q} p]}= \\
\sum_{k=0}^{b} \sum_{n=0}^{\infty} \sum_{r, s=0}^{r+s \leq n}(-1)^{k+r+s+n}\left(\begin{array}{l}
b \\
k
\end{array}\right) \frac{1}{r ! s !(n-r-s) !} t^{n} x^{n} \bar{q}^{r} q^{a+k+s} \bar{p}^{n-r} p^{b-k+n-s} .
\end{gathered}
$$

The terms that must be subtracted in the minimal scheme are determined by the inequality

$$
b-k+2 n-r-s \leq a+b-2,
$$

from which, upon use of $r+s \leq n$, the inequality $a+k+s>r$ follows. All the subtraction terms have therefore the following $q$ dependence

$$
q^{y}(q \bar{q})^{x}, \quad \text { with } \quad y>0
$$


i.e. they always contain extra factors $q$, not paired with $\bar{q}$. The renormalized, minimally subtracted integral (A.3) becomes then

$$
I_{a b}^{R}(p, m)=\int \frac{\mathrm{d}^{2} q}{(2 \pi)^{2}} \int_{0}^{\infty} \mathrm{d} t t \int_{0}^{1} \mathrm{~d} x e^{-t\left(q \bar{q}+m^{2}\right)}\left[q^{a}(p-q)^{b} e^{-t x[p \bar{p}-q \bar{p}-\bar{q} p]}-\sum^{\prime} a_{r, s} q^{y}(q \bar{q})^{x} p^{r} \bar{p}^{s}\right],
$$

where $\sum^{\prime}$ stands for the sum restricted to the terms satisfying the conditions $r+s \leq a+b-2$, $y>0$. Treated separately, the terms in the integrand of (A.5) may be UV divergent, but keeping them together the integral is by construction UV convergent. The mass $m$ ensures IR convergence (the region $t \rightarrow \infty$ ) as well. In conclusion, (A.5) is an absolutely convergent multiple integral on which manipulations like changes of variables, interchanges in the order of the integration, etc, are mathematically well-defined, provided the integrand is treated as a whole.

Now we use this freedom, and first perform the integral over $q$. Due to the general form (A.4) of the subtraction terms and the general result

$$
\int \frac{\mathrm{d}^{2} q}{(2 \pi)^{2}} e^{-q \bar{q}} q^{n}=0
$$

none of them survives the $q$ integration, yielding

$$
I_{a b}^{R}(p, m)=\int_{0}^{1} d x \int_{0}^{\infty} d t t \int \frac{\mathrm{d}^{2} q}{(2 \pi)^{2}} q^{a}(p-q)^{b} e^{-t[(q-x p)(\bar{q}-x \bar{p})+M(x)]},
$$

with $M(x)=m^{2}+x(1-x) p \bar{p}$ and where now the order of integration is fixed. The net effect of the subtraction terms has been that they have imposed a fixed integration order. Changing the loop momentum integration variable to $k=q-x p$ and using (A.6), brings (A.7) in the simpler form

$$
I_{a b}^{R}(p, m)=\int_{0}^{1} d x \int_{0}^{\infty} d t t \int \frac{\mathrm{d}^{2} k}{(2 \pi)^{2}} e^{-t k \bar{k}} e^{-t M(x)} x^{a}(1-x)^{b} p^{a+b},
$$

which after trivial (Wick rotated) $k$ and $t$ integration (in this order) results in

$$
I_{a b}^{R}(p, m)=\frac{-i}{2 \pi} p^{a+b} \int_{0}^{1} d x \frac{x^{a}(1-x)^{b}}{m^{2}+x(1-x) p \bar{p}}=I_{a b}^{R}(p, 0) F_{a b}\left(\frac{m^{2}}{p \bar{p}}\right),
$$

with $F_{a b}(0)=1$. The massless limit provides the desired result

$$
I_{a b}^{R}(p, 0)=\frac{-i}{2 \pi} \frac{p^{a+b-1}}{\bar{p}} \int_{0}^{1} \mathrm{~d} x x^{a-1}(1-x)^{b-1}=\frac{-i}{2 \pi} \frac{p^{a+b-1}}{\bar{p}} B(a, b),
$$

in terms of the well-known Euler Beta function $B(a, b)$. Expression (A.9) is the general expression for the minimally subtracted one-loop integrals needed in our computations.

\section{B Appendix: The complete one-loop anomaly in $W_{3}$}

In this appendix, the computation of the $W_{3}$ one-loop anomaly is completed, by analyzing all the possible double contractions between $R^{A}(x)$ and $R^{B}(y)$ in eq. (5.9). The analysis is performed antifield by antifield, where the antifield in question determines the $R^{A}(x)$ to be used on the lhs of (5.9) and the " $\Phi_{A}^{*}$-anomaly" or coefficient $F^{A}$ of the one-loop anomaly that is determined. The $b^{*}$-anomaly is computed in sec. 6.2. 


\section{$u^{*}$-anomaly}

The BRST transformation for $u, R^{u}=[2(\partial c) u-c(\partial u)]$, can not be contracted twice with any other quantity in the $R^{A}$ 's: no terms with both a $b$ and a $v$ antighost are contained in them. Consequently, no term proportional to $u^{*}$ arises in the anomaly.

\section{$c^{*}$-anomaly}

The only term in the BRST transformation $R^{c}$ of $c$ that can give double contractions with other terms in $R^{B}$ is the monomial $2 k T(\partial u) u$. Moreover, this always goes via the matter loops studied for the $b^{*}$-anomaly in the main text. The direct consequence of this simple fact is that all the Ward identities that hold for the matter-loop induced part of the $b^{*}$-anomaly will hold as well upon replacing the antifield $b^{*}$ with the combination $-2 k c^{*}(\partial u) u$. In fact, this amounts to rewriting the relevant interaction term $c^{*}[2 k T(\partial u) u]$ in $(6.2)$ as the product of an "effective" antifield $\tilde{h}^{*}=-2 k c^{*}(\partial u) u$, with exactly the same quantum numbers as $b^{*}$, times minus the matter energy momentum tensor $T$. It is then clear that the $c^{*}$-anomaly is, in analogy with (6.9)

$$
\mathcal{A}_{1, \mathrm{~m}}^{c^{*}}=\frac{i}{24 \pi} \int \mathrm{d}^{2} x c^{i j} \partial^{3}\left[2 k c^{*} u(\partial u) \delta_{i j}\right],
$$

with $c^{i j}$ defined in (6.10). The same argument allows a simple determination of the contributions to the $\phi^{*}$ and $v^{*}$ anomalies induced by matter loops as well.

\section{$\phi^{*}$-anomaly}

In the BRST transformation for the matter fields

$$
R^{\phi^{i}}=u d_{j k}^{i}\left(\partial \phi^{j}\right)\left(\partial \phi^{k}\right)+c\left(\partial \phi^{i}\right)-2 k b(\partial u) u\left(\partial \phi^{i}\right)
$$

the first monomial, can only produce contributions via matter loops, in analogy with the $c^{*}$ anomaly. Substituting $b^{*} \delta_{i j}$ in (6.9) by the effective antifield $-2 u \phi_{k}^{*} d_{i j k}$ leads then to the following matter induced contribution to the $\phi^{*}$ anomaly

$$
\mathcal{A}_{1, \mathrm{~m}}^{\phi^{*}}=\frac{i}{24 \pi} \int \mathrm{d}^{2} x c^{i j} \partial^{3}\left(-2 u \phi_{k}^{*} d_{i j k}\right) .
$$

The remaining two terms in (B.1) generate contributions to the $\phi^{*}$ anomaly through mixed matter-ghost loops or by purely ghost loops. Indeed, the monomial $c\left(\partial \phi^{i}\right)$ gives rise to mixed matter-ghost loops through contractions with the terms

$$
\left\{\begin{array}{cc}
-2 k b(\partial u) u\left(\partial \phi^{i}\right) & \text { in } \quad R^{\phi} \\
2 k T b(\partial u)+2 k \partial(T b u) & \text { in } \quad R^{v}
\end{array}\right.
$$

while the monomial $-2 k b(\partial u) u\left(\partial \phi^{i}\right)$ produces also mixed matter-ghost loops through contractions with the term $c\left(\partial \phi^{i}\right)$ in $R^{\phi}$ and purely ghost loops through contractions with the term $3 v(\partial c)+(\partial v) c$ in $R^{v}$. A good test product of fields for all these four possibilities is $\phi^{j}(y) v(z) v(t)$, which forces the corresponding anomaly to contain one $\phi$ field, two $u$ fields and four derivatives $\partial$. 
The one-loop anomalous Ward identity (5.9) takes the form

$$
\begin{gathered}
\frac{i}{\hbar}\left\langle\mathrm{N}_{0}\left[c\left(\partial \phi^{i}\right)(x)\right] \mathrm{N}_{0}\left[-2 k b(\partial u) u\left(\partial \phi^{j}\right)(y)\right] v(z) v(t)\right\rangle_{c, 0} \\
-\frac{i}{\hbar}\left\langle\mathrm{N}_{0}\left[c\left(\partial \phi^{i}\right)(x)\right] \mathrm{N}_{3}[2 k T b(\partial u)(z)+2 k \partial(T b u)(z)] v(t) \phi^{j}(y)\right\rangle_{c, 0}-(z, t) \\
+\frac{i}{\hbar}\left\langle\mathrm{N}_{0}\left[-2 k b(\partial u) u\left(\partial \phi^{i}\right)(x)\right] \mathrm{N}_{0}\left[c\left(\partial \phi^{j}\right)(y)\right] v(z) v(t)\right\rangle_{c, 0} \\
-\frac{i}{\hbar}\left\langle\mathrm{N}_{0}\left[-2 k b(\partial u) u\left(\partial \phi^{i}\right)(x)\right] \mathrm{N}_{3}[3 v(\partial c)(z)+(\partial v) c(z)] v(t) \phi^{j}(y)\right\rangle_{c, 0}-(z, t) \\
=-\alpha_{5}\left\langle\mathrm{~N}_{0}\left[F^{i}\left(\phi, u, u, \partial^{4} ; x\right)\right] \phi^{j}(y) v(z) v(t)\right\rangle_{c, 0} .
\end{gathered}
$$

After a lengthy but straightforward calculation, the lhs is found to be

$$
\begin{gathered}
\frac{k \hbar^{3}}{\pi} \int \frac{\mathrm{d}^{2} l}{(2 \pi)^{2}} \int \frac{\mathrm{d}^{2} q}{(2 \pi)^{2}} \int \frac{\mathrm{d}^{2} r}{(2 \pi)^{2}} e^{i(q+l+r) \cdot x} e^{-i q \cdot y} e^{-i r \cdot t} e^{-i l \cdot z} \\
\times \frac{1}{\bar{q} \bar{l} \bar{r}}\left[-\frac{1}{2}(r-l)(l+q+r)^{2}-\frac{1}{2}(r-l) q^{2}+2(r-l) r l+\frac{5}{6}\left(l^{3}-r^{3}\right)\right] \delta^{i j},
\end{gathered}
$$

where the factor $1 / \bar{q}$ corresponds to a propagator between $\partial \phi$ and $\phi$ and the factors $1 / \bar{r}$ and $1 / \bar{l}$ are generated by $(u, v)$ ghost propagators. The combinations of momenta in (B.2) can be associated to the following monomials

$$
\begin{cases}l^{3}-r^{3} & \longleftrightarrow u\left(\partial^{3} u\right)(\partial \phi) \\ r^{2} l-l^{2} r & \longleftrightarrow(\partial u)\left(\partial^{2} u\right)(\partial \phi) \\ (r-l) q^{2} & \longleftrightarrow u(\partial u)\left(\partial^{3} \phi\right) \\ (r-l)(l+q+r)^{2} & \longleftrightarrow \partial^{2}(u(\partial u)(\partial \phi)) .\end{cases}
$$

After computing the coefficients of the different terms in the usual way and performing a few integrations by parts, the second contribution to the $\phi^{*}$ anomaly is

$$
\mathcal{A}_{1, \mathrm{gh}}^{\phi^{*}}=\frac{-i k}{6 \pi} \int \mathrm{d}^{2} x \phi_{i}^{*}\left[6 \partial\left(u(\partial u)\left(\partial^{2} \phi^{i}\right)\right)+9\left(\partial^{2} u\right)(\partial u)\left(\partial \phi^{i}\right)+8 u\left(\partial^{3} u\right)\left(\partial \phi^{i}\right)\right] .
$$

\section{$v^{*}$-anomaly}

Let us finally compute the contribution to the one-loop anomaly induced by the BRST transformation of the ghost field $v$

$$
R^{v}=-W+2 k T b(\partial u)+2 k \partial(T b u)+3 v(\partial c)+(\partial v) c .
$$

The contributions caused by matter loops are generated by double contractions between matter fields in $-W$ or in the energy-momentum tensor $T$ in (B.4) with $(\partial \phi)^{2}$ type terms in the $R^{B}$ 's. When writing the interaction terms in the form of effective sources times $-T$ in analogy with the matter induced $b^{*}$-anomaly, the following matter induced contribution the one-loop $v^{*}$-anomaly is inferred

$$
\mathcal{A}_{1, \mathrm{~m}}^{v^{*}}=\frac{i}{24 \pi} \int \mathrm{d}^{2} x c^{i j} \partial^{3}\left[2 v^{*} d_{i j k}\left(\partial \phi^{k}\right)+2 k b\left(u \partial v^{*}-v^{*} \partial u\right) \delta_{i j}\right] .
$$

The computation of the contribution of the loops with at least one ghost line, requires more effort. Closed loops of one matter field and one ghost or of two ghosts can be obtained in the following four ways. The terms $[2 k T b(\partial u)+2 k \partial(T b u)]$ get contracted twice with

$$
\left\{\begin{array}{ccc}
3 v(\partial c)+(\partial v) c & \text { in } & R^{v} \\
c\left(\partial \phi^{i}\right) & \text { in } & R^{\phi}
\end{array}\right.
$$


while the terms $[3 v(\partial c)+(\partial v) c]$ can contract with

$$
\left\{\begin{array}{clc}
-2 k b(\partial u) u\left(\partial \phi^{i}\right) & \text { in } & R^{\phi} \\
2 k T b(\partial u)+2 k \partial(T b u) & \text { in } & R^{v}
\end{array}\right.
$$

A suitable testproduct for all four contributions is $\phi^{j}(y) \phi^{k}(z) v(t)$, yielding a contribution to the $v^{*}$-anomaly constructed out of two $\phi$-fields, one $u$ ghost and five derivatives $\partial$, and a one-loop anomalous Ward identity of the form

$$
\begin{gathered}
-\frac{i}{\hbar}\left\langle\mathrm{N}_{3}[2 k T b(\partial u)(x)+2 k \partial(T b u)(x)] \mathrm{N}_{3}[3 v(\partial c)(t)+(\partial v) c(t)] \phi^{j}(y) \phi^{k}(z)\right\rangle_{c, 0} \\
+\frac{i}{\hbar}\left\langle\mathrm{N}_{3}[2 k T b(\partial u)(x)+2 k \partial(T b u)(x)] \mathrm{N}_{0}\left[c\left(\partial \phi^{j}\right)(y)\right] \phi^{k}(z) v(t)\right\rangle_{c, 0}+(j, k)(y, z) \\
+\frac{i}{\hbar}\left\langle\mathrm{N}_{3}[3 v(\partial c)(x)+(\partial v) c(x)] \mathrm{N}_{0}\left[-2 k b(\partial u) u\left(\partial \phi^{j}\right)(y)\right] \phi^{k}(z) v(t)\right\rangle_{c, 0}+(j, k)(y, z) \\
-\frac{i}{\hbar}\left\langle\mathrm{N}_{3}[3 v(\partial c)(x)+(\partial v) c(x)] \mathrm{N}_{3}[2 k T b(\partial u)(t)+2 k \partial(T b u)(t)] \phi^{j}(y) \phi^{k}(z)\right\rangle_{c, 0} \\
=-\alpha_{6,7}\left\langle\mathrm{~N}_{3}\left[F\left(u, \phi, \phi, \partial^{5} ; x\right)\right] \phi^{j}(y) \phi^{k}(z) v(t)\right\rangle_{c, 0} .
\end{gathered}
$$

The equality of the first and fourth contribution on the lhs of (B.5) upon exchanging $x$ and $t$, and the fact that the value of the second and third contributions can be read off from some of the results obtained in the $\phi^{*}$ - computation above, reduce the amount of work necessary to obtain the renormalized expresion for the lhs of $(\mathrm{B} .5)$. It reads

$$
\begin{gathered}
\frac{-k \hbar^{3}}{\pi} \int \frac{\mathrm{d}^{2} l}{(2 \pi)^{2}} \int \frac{\mathrm{d}^{2} q}{(2 \pi)^{2}} \int \frac{\mathrm{d}^{2} r}{(2 \pi)^{2}} e^{i(q+l+r) \cdot x} e^{-i q \cdot y} e^{-i r \cdot t} e^{-i l \cdot z} \\
\frac{1}{\bar{q} \bar{l} \bar{r}}\left[\frac{1}{2}\left(r\left(l^{2}+q^{2}\right)+(l+q+r)\left(l^{2}+q^{2}\right)\right)\right. \\
\left.+\frac{1}{6}\left(5 r^{3}+12 r^{2}(l+q+r)+12 r(l+q+r)^{2}+5(l+q+r)^{3}\right)\right] .
\end{gathered}
$$

It is now a piece of cake to determine the distribution of the five derivatives $\partial$ in the coefficients $F\left(u, \phi, \phi, \partial^{5} ; x\right)$. Indeed, taking into account that the factor $(\bar{q} \bar{l} \bar{r})^{-1}$ is generated by two propagators between $\phi$ and $\partial \phi$, and one propagator $(u, v)$, the other momentum functions can be associated with the following terms in $F\left(u, \phi, \phi, \partial^{5} ; x\right)$

$$
\begin{cases}r\left(l^{2}+q^{2}\right) & \longleftrightarrow(\partial \phi)\left(\partial^{3} \phi\right) \partial u \\ (r+l+q)\left(l^{2}+q^{2}\right) & \longleftrightarrow \partial\left(u(\partial \phi)\left(\partial^{3} \phi\right)\right) \\ r^{3} & \longleftrightarrow\left(\partial^{3} u\right)(\partial \phi)(\partial \phi) \\ r^{2}(r+l+q) & \longleftrightarrow \partial\left(\left(\partial^{2} u\right)(\partial \phi)(\partial \phi)\right) \\ r(r+l+q)^{2} & \longleftrightarrow \partial^{2}((\partial u)(\partial \phi)(\partial \phi)) \\ (r+l+q)^{3} & \longleftrightarrow \partial^{3}(u(\partial \phi)(\partial \phi)) .\end{cases}
$$

By simple comparison with (B.6), the coefficients of each of these terms are determined and the mixed and ghost induced contributions to the one-loop $v^{*}$-anomaly is

$$
\begin{aligned}
\mathcal{A}_{1, \mathrm{gh}}^{v^{*}}= & \frac{i k}{2 \pi} \int \mathrm{d}^{2} x\left(v^{*}(\partial u)-u\left(\partial v^{*}\right)\right)\left(\partial \phi^{i}\right)\left(\partial^{3} \phi^{i}\right) \\
& +\frac{i k}{6 \pi} \int \mathrm{d}^{2} x T\left[5\left(\partial^{3} u\right) v^{*}-12\left(\partial^{2} u\right)\left(\partial v^{*}\right)+12(\partial u)\left(\partial^{2} v^{*}\right)-5 u\left(\partial^{3} v^{*}\right)\right] .
\end{aligned}
$$


In summary, collecting all the previous contributions, we can write the complete one-loop anomaly for $W_{3}$ in the compact way (6.11). This finishes our analysis of the one-loop contribution to the BRST anomaly in the $W_{3}$ model.

\section{The ghost number anomaly}

In this appendix, we show how the well-known ghost number anomaly [45] is obtained in the context of the BPHZ scheme. Although in principle this is another application of the method sketched in sect. 3.4 for computing the global axial anomaly, we find it instructive to present it as an example of how nonlocal mass terms, though invariant, may give rise to anomalous conservation laws. Also, the fact that the correct ghost number anomaly is obtained should dispell any misgivings about the use of this non-local mass-term.

In order to make contact with standard formulations of chiral gravities, we consider a simplified version of the models used before, with all antifields set to zero except the antifield $b^{*}$, which takes over the rôle of (minus) the gravitational gauge field, i.e.

$$
\tilde{S}=\int \mathrm{d}^{2} x\left[-\frac{1}{2}(\partial \phi)(\bar{\partial} \phi)+b_{j}\left(\bar{\partial} c_{j}\right)-\frac{m^{2}}{2}\left(\phi^{2}+2 b_{j} \frac{1}{\partial} c_{j}\right)-b^{*}\left[T+T_{2}+T_{3}\right]\right] .
$$

$\left\{c_{j}, b_{j}\right\}, j=2,3$ are the spin 2 and 3 ghost-antighosts systems and $T_{2}, T_{3}$ their corresponding energy momentum tensors (5.3). As it stands, this massive action (C.1) is invariant under the following ghost number transformations

$$
\delta_{\sharp} \Phi^{A}=\sharp_{A} \Phi^{A}, \quad \delta_{\sharp} b^{*}=0,
$$

where the ghost numbers $\sharp_{A}$ for the fields are listed in tables 1 and 2 . One would naively conclude that there is no room for a ghost number anomaly in our formulation, contrary to the well-known results. However, this is not the case and, once again, the oversubtractions performed on the IR regulating mass term, together with its nonlocal character, are the origin of the ghost number anomaly.

As usual for global symmetries, the analysis starts by computing the divergence of the ghost number current $(S=\tilde{S}(m=0))$

$$
\partial_{\mu} j^{\mu}(x)=-\left[\frac{\overleftarrow{\delta} S}{\delta \Phi^{A}(x)} \delta_{\sharp} \Phi^{A}(x)\right]+m^{2} \sum_{j=2,3}\left[\left(\frac{1}{\partial} b_{j}\right) c_{j}+b_{j}\left(\frac{1}{\partial} c_{j}\right)\right]
$$

which classically vanishes on-shell in the limit $m \rightarrow 0$. The anomalous Ward identity is

$$
\begin{gathered}
\left\langle\mathrm{N}_{2}\left[\partial_{\mu} j^{\mu}(x)\right] \prod_{i} \Phi^{A_{i}}\left(x_{i}\right)\right\rangle_{c}= \\
-i \hbar \sum_{i} \sharp A_{i}\left\langle\prod_{i} \Phi^{A_{i}}\left(x_{i}\right)\right\rangle_{c} \delta\left(x-x_{i}\right)+\left\langle\mathrm{N}_{2}[\mathcal{G}(x)] \prod_{i} \Phi^{A_{i}}\left(x_{i}\right)\right\rangle_{c},
\end{gathered}
$$

where the operator insertion $\mathcal{G}(x)$ parametrises the ghost number anomaly. The linearity of the transformations (C.2) allows, upon application of the Schwinger-Dyson equations (3.5), to rewrite the contribution from the massless piece in (C.3) as

$$
\left\langle\mathrm{N}_{2}\left[-\left(\frac{\overleftarrow{\delta} S}{\delta \Phi^{A}(x)} \delta_{\sharp} \Phi^{A}(x)\right)\right] \prod_{i} \Phi^{A_{i}}\left(x_{i}\right)\right\rangle_{c}=-i \hbar\left\langle\left(\delta_{\sharp} \Phi^{A}(x) \frac{\vec{\delta}}{\delta \Phi^{A}(x)}\right) \prod_{i} \Phi^{A_{i}}\left(x_{i}\right)\right\rangle_{c}
$$


which reproduces the first, non-anomalous term on the rhs of the Ward identity (C.4). The ghost number anomaly is therefore determined by the oversubtracted operator

$$
m^{2} \sum_{j=2,3} \mathrm{~N}_{2}\left[\left(\frac{1}{\partial} b_{j}\right) c_{j}(x)+b_{j}\left(\frac{1}{\partial} c_{j}(x)\right)\right] .
$$

No anisotropic normal orderings appear because the transformation rules of the ghost number symmetry are linear.

Once again, prior to taking the limit $m \rightarrow 0$, the Zimmerman identity (3.7) should be used

$$
m^{2} \sum_{j=2,3}\left\{\mathrm{~N}_{2}\left[\left(\frac{1}{\partial} b_{j}\right) c_{j}+b_{j}\left(\frac{1}{\partial} c_{j}\right)\right]-\mathrm{N}_{0}\left[\left(\frac{1}{\partial} b_{j}\right) c_{j}+b_{j}\left(\frac{1}{\partial} c_{j}\right)\right]\right\}=\sum_{k} r_{k} \mathrm{~N}_{2}\left[\mathcal{G}_{k}\right] .
$$

Its rhs is determined in the usual way by inserting both sides of this relation in suitable correlation functions. In doing this computation, however, some work can be saved by using the dimensional arguments presented in sect. 5.2. They show that the insertions $\mathcal{G}_{k}(x)$ in (C.5) must be of the form $\mathcal{G}_{k} \sim G(\partial) b^{*}$. In this way, it is concluded that the one-loop contributions to the correlation functions

$$
\begin{gathered}
M_{a}^{j}(x)=\frac{i}{\hbar} \int \mathrm{d}^{2} y b^{*}(y) I_{a}^{j}(x-y)= \\
\frac{i}{\hbar} \int \mathrm{d}^{2} y b^{*}(y)\left\langle\mathrm{N}_{2}\left[j b_{j}\left(\partial c_{j}\right)+(j-1)\left(\partial b_{j}\right) c_{j}\right](y) \mathrm{N}_{a}\left[\left(\frac{1}{\partial} b_{j}\right) c_{j}+b_{j}\left(\frac{1}{\partial} c_{j}\right)\right]\right\rangle_{c, 0},
\end{gathered}
$$

given in terms of the BPHZ renormalized one-loop integrals

$$
I_{a}^{j}(x-y)=-\hbar^{2} \int \frac{\mathrm{d}^{2} p}{(2 \pi)^{2}} e^{i p \cdot(x-y)} \int \frac{\mathrm{d}^{2} q}{(2 \pi)^{2}}\left(1-t_{p}^{a}\right)\left[\frac{p q+(j-1) p^{2}}{\left(q \bar{q}+m^{2}\right)\left[(p-q)(\bar{p}-\bar{q})+m^{2}\right]}\right],
$$

provide the relevant information on what the rhs of (C.5) exactly is in terms of the difference $\sum_{j=2,3}\left(M_{2}^{j}(x)-M_{0}^{j}(x)\right)$.

Computing the difference $I_{2}^{j}(x-y)-I_{0}^{j}(x-y)$ requires the knowledge of the $p$ expansion of the integrand in (C.6) up to second order. Keeping only terms of the form $(q \bar{q})^{n}$ in the Taylor series (see appendix A), we obtain

$$
\begin{gathered}
I_{2}^{j}(x-y)-I_{0}^{j}(x-y)= \\
\hbar^{2} \int \frac{\mathrm{d}^{2} p}{(2 \pi)^{2}} e^{i p \cdot(x-y)} \int \frac{\mathrm{d}^{2} q}{(2 \pi)^{2}} t_{p}^{2}\left[\frac{p q+(j-1) p^{2}}{\left(q \bar{q}+m^{2}\right)\left[(p-q)(\bar{p}-\bar{q})+m^{2}\right]}\right]= \\
-\hbar^{2} \int \frac{\mathrm{d}^{2} p}{(2 \pi)^{2}} e^{i p \cdot(x-y)} p^{2} \int \frac{\mathrm{d}^{2} q}{(2 \pi)^{2}}\left[\frac{(j-1)}{\left(q \bar{q}+m^{2}\right)^{2}}+\frac{q \bar{q}}{\left(q \bar{q}+m^{2}\right)^{3}}\right]= \\
\frac{i(2 j-1) \hbar^{2}}{4 \pi m^{2}} \int \frac{\mathrm{d}^{2} p}{(2 \pi)^{2}} e^{i p \cdot(x-y)} p^{2}=-\frac{i(2 j-1) \hbar^{2}}{4 \pi m^{2}} \partial^{2} \delta(x-y) .
\end{gathered}
$$

Plugging this result back in the difference $\sum_{j=2,3}\left(M_{2}^{j}(x)-M_{0}^{j}(x)\right)$, we easily obtain

$$
M_{2}^{j}(x)-M_{0}^{j}(x)=\frac{(2 j-1) \hbar}{4 \pi m^{2}} \partial^{2} b^{*}(x) .
$$

The Zimmerman identity (C.5) then takes the form

$$
m^{2} \sum_{j=2,3}\left\{\mathrm{~N}_{2}\left[\left(\frac{1}{\partial} b_{j}\right) c_{j}+b_{j}\left(\frac{1}{\partial} c_{j}\right)\right]-\mathrm{N}_{0}\left[\left(\frac{1}{\partial} b_{j}\right) c_{j}+b_{j}\left(\frac{1}{\partial} c_{j}\right)\right]\right\}=\frac{(3+5) \hbar}{4 \pi} \partial^{2} b^{*}
$$


from which, upon taking the limit $m \rightarrow 0$, the expression of the anomalous insertion in (C.4) is finally determined

$$
\mathcal{G}(x)=\frac{2 \hbar}{\pi} \partial^{2} b^{*}(x)
$$

This is in agreement with previous computations in the literature [45].

We conclude that, despite the invariance of the mass term, the correct anomalous Ward identity is obtained. The anomaly arises from oversubtracted operators which occur with a coefficient proportional to the mass (squared). The massless limit of these combinations is extracted using Zimmerman's normal product identity. 


\section{References}

[1] C. BECCHI, An introduction to the renormalization of theories with continuous symmetries, to the chiral models and to their anomalies, Lectures given at the 29th Int. Universitatswochen fur Kernphysik 1990, Schladming, Austria, March 1990. Schladming School, 1990; pp. 31-52.

[2] For an early review, using the BPHZ renormalization scheme also used in this paper, see O. Piguet And A. Rouet, Phys. Rep. 76 (1981) 1.

[3] I. A. Batalin and G. A. Vilkovisky, Phys. Lett. B102 (1981) 27; ibid. 120B (1983) 166; Phys. Rev. D28 (1983) 2567; [E: D30 (1984) 508].

[4] W. Troost, P. van Nieuwenhuizen and A. Van Proeyen, Nucl. Phys. B333 (1990) 727.

[5] J. PArís, Nucl. Phys. B450 (1995) 357.

[6] J. París and W. Troost, "Higher Loop Anomalies and their Consistency Conditions in Nonlocal Regularization"; preprint KUL-TF-96/5.

[7] M. Tonin, Nucl. Phys. (Proc. Suppl.) B29 (1992) 137.

[8] S. Adler, Phys. Rev. 177 (1969) 2426;

W. A. Bardeen, Phys. Rev. 184 (1969) 1848;

J. S. Bell AND R. JaCkiW, Nuovo Cim. 60A (1969) 47.

[9] J.H. Löwenstein, BPHZ renormalization, in Renormalization Theory, eds. G. Velo and A.S. Wightman, Proceedings of the NATO ASI, Erice 1975.

[10] G.'т Hooft and M. Veltman, Nucl. Phys. B44 (1972) 189.

[11] O. Piguet and S. P. Sorella, Algebraic Renormalization: Perturbative Renormalization, Symmetries and Anomalies, Lecture Notes in Physics vol. 28, Springer 1995.

[12] J. Gomis, J. París and S. Samuel, Phys. Rep. 259 (1995) 1.

[13] W. Troost and A. Van Proeyen, An introduction to Batalin-Vilkovisky Lagrangian Quantisation, Leuven Notes in Math. Theor. Phys., in preparation.

[14] F. DE Jonghe, The Batalin-Vilkovisky Lagrangian quantisation scheme with applications to the study of anomalies in gauge theories, Ph. D. thesis, K. U. Leuven, 1994, hep-th/9403143.

[15] M. Henneaux, Nucl. Phys. B18A (1990) 47 (Proc. Suppl.);

M. Henneaux and C. Teitelboim, Quantisation of gauge systems, Princeton University Press, 1992.

[16] C. Becchi, A. Rouet and R. Stora, Phys. Lett. B52 (1974) 344; Commun. Math. Phys. 42 (1975) 127; Ann. Phys. (N.Y.) 98 (1976) 287.

[17] B. De Wit and J. W. van Holten, Phys. Lett. B79 (1978) 389.

[18] J. Zinn-Justin, in Trends in Elementary Particle Theory, Lecture notes in Physics 37, Int. Summer Inst. on Theor. Phys., Bonn 1974, eds. H. Rollnik and K. Dietz, Springer, Berlin, 1975; Nucl. Phys. B246 (1984) 246; and Quantum Field Theory and Critical Phenomena, Oxford University Press, 1989. 
[19] I. A. Batalin and G. A. Vilkovisky, J. Math. Phys. 26 (1985) 172.

[20] M. Henneaux, Commun. Math. Phys. 140 (1991) 1.

[21] S. Vandoren and A. Van Proeyen, Nucl. Phys. B411 (1994) 257.

[22] Y. M. P. Lam, Phys. Rev. D6 (1972) 2145, 2161.

[23] T. E. Clark and J. H. Löwenstein, Nucl.Phys. B113 (1976) 109.

[24] P. S. Howe, U. Lindström And P. White, Phys. Lett. B246 (1990) 430.

[25] D. Anselmi, Class. Quantum Grav. 11 (1994) 2181.

[26] J. Gomis And S. WeinberG, "Are nonrenormalizable gauge theories renormalizable?" preprint RIMS-1036, October 1995; hep-th/9510087.

[27] K. Fujikawa, Phys. Rev. Lett. 42 (1979) 1195; Phys. Rev. D21 (1980) 2848; Phys. Rev. D25 (1982) 2584.

[28] A. Diaz, W. Troost, P. van Nieuwenhuizen and A. Van Proeyen, Int. J. Mod. Phys. A4 (1989) 3959.

[29] L. D. Faddeev and A. A. Slavnov, Gauge Fields: Introduction to Quantum Theory, (Benjamin-Cummings, Reading, MA, 1980).

[30] C. P. Martin and F. Ruiz Ruiz, Nucl. Phys. B436 (1995) 545;

M. Asorey and F. Falceto, "On the consistency of the Regularization of Gauge Theories by High Covariant Derivatives", preprint DFTUZ-95.3; hep-th/9502025;

T. D. Bakeyev and A. A. Slavnov, "Higher Covariant Derivative Regularization Revisited", preprint SMI-02-96; hep-th/9601092;

P. Claus, "Higher covariant derivative Pauli-Villars regularization for gauge theories in the Batalin-Vilkovisky formalism", Leuven preprint KUL-TF-96/1; hep-th/9601095.

[31] D. Evens, J. W. Moffat, G. Kleppe and R. P. Woodard, Phys. Rev. D43 (1991) 499; G. Kleppe and R. P. Woodard, Nucl. Phys. B388 (1992) 81; Ann. Phys. (N.Y.) 221 (1993) 106.

[32] P. Breitenlohner and D. Maison, Commun. Math. Phys. 52 (1977) 11, 39, 55.

[33] N. N. Bogoliubov And D. W. Shirkov, Introduction to the Theory of Quantized Fields, Interscience Publ., New York, 1959;

N. N. Bogoliubov and O. Parasiuk, Acta Math. 97 (1957) 227;

K. Hepp, Commun. Math. Phys. 2 (1966) 301;

[34] W. Zimmerman, Commun. Math.Phys. 15 (1969) 208; Ann. Phys. (N.Y.) 77 (1973) 570.

For a pedagogical account, which can be viewed as prerequisite knowledge for [9], see W. Zimmerman, in Lectures on Elementary Particles and Quantum Field Theory, eds. S. Deser, M. Grisaru and H. Pendleton, MIT Press, Brandeis Lectures 1970. A more modern account, set in the BRS language and also including the necessary information on generating functionals used in section [4, is [11].

[35] M. Gomes and J. H. Lowenstein, Phys. Rev. D7 (1973) 550. 
[36] F. De Jonghe, R. Siebelink en W. Troost, Phys. Lett. B288 (1992) 47; B306 (1993) 295.

[37] C. M. Hull, Phys. Lett. B240 (1990) 110.

[38] Y. Matsuo, Phys. Lett. B227 (1989) 222; in Proc. of the meeting Geometry and Physics, Lake Tahoe, July 1989.

[39] C. M. Hull, Phys. Lett. B265 (1991) 347.

[40] K. Schoutens, A. Sevrin and P. van Nieuwenhuizen, Nucl. Phys. B364 (1991) 584; in Proc. of the January 1991 Miami workshop on Quantum Field Theory, Statistical Mechanics, Quantum Groups and Topology.

[41] C. N. Pope, L. J. Romans And K. S. Stelle, Phys. Lett. B268 (1991) 167.

[42] C. M. Hull, Int. J. Mod. Phys. A8 (1993) 2419.

[43] R. Mohayaee and L. Wenham, Nucl. Phys. B454 (1995) 207.

[44] F. De Jonghe, R. Siebelink, W. Troost, S. Vandoren, P. van Nieuwenhuizen and A. Van Proeyen, Phys. Lett. B289 (1992) 354.

[45] K. Fujikawa, Phys. Rev. D25 (1982) 2584. 